\title{
基于氧杂葸结构可控官能化的荧光探针研究新进展
}

\author{
黄阳阳王梦嘉厍梦尧杨征 \\ 刘萍李剑利* 史真 \\ (西北大学化学与材料科学学院 合成与天然功能分子化学教育部重点实验室 西安 710069)
}

\begin{abstract}
摘要 荧光分子探针技术在表达分子间识别行为及复杂生命和环境体系的内状态信息方面具有非常优异的性能, 氧杂 葱及其衍生物螺连隐色体结构变化伴随的分子苂光变换模型, 广泛且深入的应用于构建新型功能光敏探针分子. 近年 来此类探针的合成设计及功能化调控研究异常活跃, 新的突破不断涌现. 综述了新近基于氧杂葱及其衍生物的荧光探 针在金属阳离子、 $\mathrm{pH}$ 、阴离子识别检测方面的研究进展, 并简要阐释了该类探针分子的构筑, 识别检测机理以及探针 在生物成像和环境监测等方面的应用.
\end{abstract}

关键词 氧杂葱; 荧光探针; 生物成像

\section{Recent Progress in the Fluorescent Probe Based on Spiro Ring Opening of Xanthenes and Related Derivatives}

\author{
Huang, Yangyang Wang, Mengjia She, Mengyao Yang, Zheng \\ Liu, Ping Li, Jianli* Shi, Zhen \\ (Key Laboratory of Synthetic and Natural Functional Molecular Chemistry, Ministry of Education, \\ College of Chemistry \& Material Science, Northwest University, Xi'an 710069)
}

\begin{abstract}
Fluorescent probe technology has specially outstanding function in the track of the heterogeneous recognition behaviors and the information from the internal status of the complicated biological and environmental systems. The spiral ring structure of xanthene and related derivatives which substrate triggered fluorescent switching is the most extensively used model in construction of novel photosensitive fluorescent probes. In recent years, design, synthesis and functional regulation and control of this type of probe are active sub-discipline with continuous breakthroughs. In this paper, the most recent advances in the detection of different metal cations, $\mathrm{pH}$ deviations, anions which based on xanthene and related derivatives are reviewed. The construction of xanthene based fluorescent probes, the recognition mechanisms and the application in bioimaging and environmental monitoring are particularly highlighted.
\end{abstract}

Keywords xanthenes; fluorescent probe; bioimaging

自然界中, 分子识别是广泛存在的一种现象, 其识 别行为所表达的信息 ${ }^{[1]}$ 往往对于重大科学问题具有先导 性. 近年来, 荧光化学标记和分子探针技术伴随着其强 劲的应用前景发展迅速, 被广泛应用在复杂生物和环境 体系的内状态信息表达、药物高通量篎选及苂光组织化 学研究等领域, 开创了众多交叉科学研究的新突破 ${ }^{[2]}$.
这一领域持续的创新推进更加丰富了苂光分子探针独 特的性能优势: 灵敏度和选择性强，可达单分子检测; 开关可控，易于实现与分子内信息的通讯；可实现对亚 微粒在亚纳米空间、亚毫秒时间内的可视化分辨; 荧光 成像的原位检测, 亦可利用光纤进行远距离检测等 ${ }^{[3]}$. 当今光敏探针技术正趋于多功能的集成，其宽广的应用

*E-mail: lijianli@nwu.edu.cn

Received August 14, 2013; revised September 10, 2013; published online September 25, 2013.

Project supported by the National Natural Science Foundation of China (Nos. 21272184, 20972124, J1210057), the Shaanxi Provincial Natural Science Fund Project (No. 2012JQ2007), the Special Science Research Foundation of Education Committee in Shaanxi Province (No. 12JK0584), the Northwest University Science Foundation for Postgraduate Students (No. YZZ12030), and the Chinese National Innovation Experiment Program for University Students (No. 201210697011).

国家自然科学基金(Nos. 21272184, 20972124, J1210057)、陕西省自然科学基础研究计划项目基金(No. 2012JQ2007)、陕西省教育厅专项计划项目基金 (No. 12JK0584)、西北大学研究生创新基金(No. YZZ12030)、国家大学生创新性计划项目基金(No. 201210697011)资助项目. 
前景源于新型探针分子的合成设计、结构调控与性能优 化.

苂光探针分子构建中荧光团是至关重要的结构片 段, 常用的荧光团有氧杂蒽、香豆素、呋啶、萠、1,8荟二甲酰亚胺、花菁和氟化硼二吡咯(BODIPY)等, 其中 氧杂蒽(主要涉及罗丹明和苂光素系列)结构单元, 因其 摩尔吸收系数高、激发和发射波长较长、菼光量子产率 高、水溶性好和适用的 $\mathrm{pH}$ 范围较宽等特点而成为该领 域的 “明星” 结构基元 ${ }^{[4]}$. 氧杂蒽结构单元的苂光探针 分子基本架构如图(Scheme 1), 其信息表达是通过间隔 基传递识别基结合客体分子所产生的分子作用信息，从 而调控荧光体底环(苯环)与顶环(氧杂蒽环)之间螺环隐 色体结构变化伴随的苂光改变.

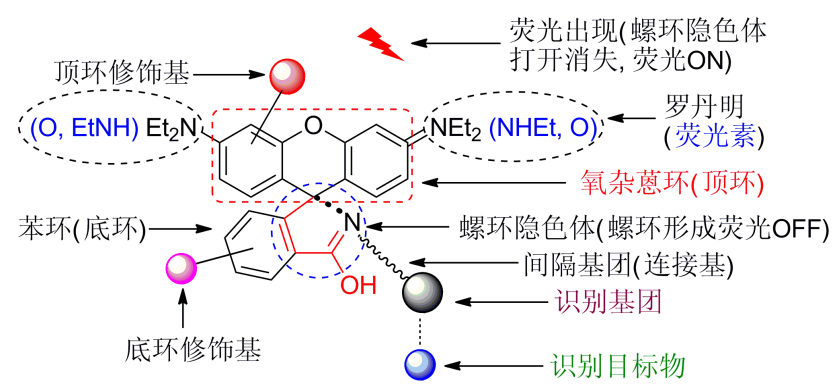

Scheme 1

氧杂葸结构探针分子中荧光团最为经典的当属罗
丹明 $\mathrm{B}$ 和荧光素及其衍生物. 通常探针分子一般都含有 一个与氧杂蒽环螺连的内酰胺或内酯结构片段，通过被 识别基团的诱导而使内酰胺或内酯片段发生开环与关 环(Scheme 2), 进而调控整个分子中苂光才氧杂葸环共 轭体系发生相应的改变(醌式体系的形成与消失), 同时 伴随着探针分子菼光的保持(ON 型)或消失(OFF 型).

1997 年, Czarnik 等 ${ }^{[5]}$ 报道了罗丹明 B 类荧光探针及 其开环反应的开创性工作，拉开了这一领域蓬勃发展的 序幕. 至此基于 Czarnik 的研究成果, 更多与之相关的 新型探针研究如雨后春笋般不断涌现 ${ }^{[6]}$. 本课题组 ${ }^{[7]}$ 也 曾于 2010 年综述了罗丹明系列 OFF-ON 苂光探针的阶 段性研究进展. 近年来氧杂葱及其衍生物结构片段的光 敏探针发展迅猛，新的突破层出不穷，本文根据被检测 物质的不同系统综述了该领域荧光探针及其衍生物的 设计合成研究的新进展，并对部分探针分子的识别检测 机理以及探针在生物成像和环境监测等方面的应用进 行了阐释。

\section{1 基于氧杂葸环的金属离子探针}

\section{$1.1 \mathrm{Ca}^{2+}$ 离子探针}

乙二醇-双-(2-氨基乙醚)四乙酸(EGTA)，钲螯合剂 水合钠盐(BAPTA)和喹啉酸-2,6-甲氧基-8-氨基喹啉 (Quin2)是目前 $\mathrm{Ca}^{2+}$ 离子荧光探针中普遍采用的离子配

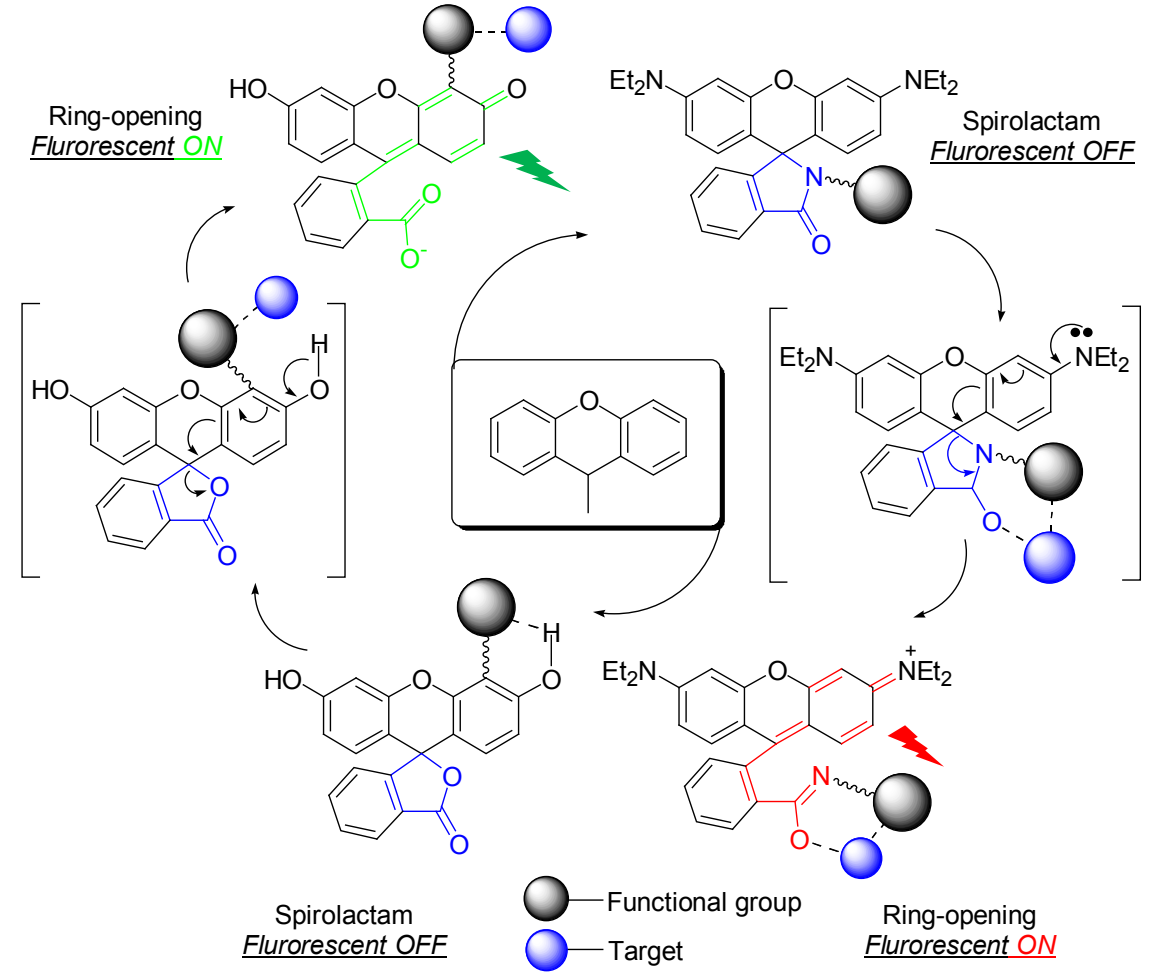

Scheme 2 
体结构. 2009 年, 我们课题组 ${ }^{[8]}$ 设计合成了 BAPTA 型的 $\mathrm{Ca}^{2+}$ 探针 1 (Eq. 1), 并对其苂光性能和生物应用性进行 了系统研究. 探针 $\mathbf{1}$ 和 $\mathrm{Ca}^{2+}$ 具有较强的结合能力, 将其 和含有 $\mathrm{Ca}^{2+}$ 的 CHO-M1 细胞共培养可观察到明显的苂 光增强现象, 表明 $\mathbf{1}$ 可很好的应用于生命体系及生物医 学中 $\mathrm{Ca}^{2+}$ 的检测.

2011 年, Marriott 等 ${ }^{[9]}$ 设计合成了一种含有 BAPTA 结构的新型 $\mathrm{Ca}^{2+}$ 探针 2 (Eq. 2). 与传统的 $\mathrm{Ca}^{2+}$ 探针相比, 2 是通过光学转换调节 BAPTA 基团的电子性能来识别 $\mathrm{Ca}^{2+}$. 探针 2 对 $\mathrm{Ca}^{2+}$ 的识别具有较高的选择性, $\mathrm{Mg}^{2+}$ 的 存在并不会对其识别产生较大干扰. 此外, 2 对 $\mathrm{Ca}^{2+}$ 的 络合作用具有可逆性, 可循环重复使用. 这也是首次报 道的可在样品中模仿细胞或组织中天然 $\mathrm{Ca}^{2+}$ 振动, 并 形成令人满意的清晰合理波形的 $\mathrm{Ca}^{2+}$ 检测方法, 显示 出良好的应用前景.

2012 年, Mallet 等 ${ }^{[10]}$ 报到了一系列含有 BAPTA 功 能基团、可用于生物样品中 $\mathrm{Ca}^{2+}$ 荧光成像的罗丹明基 $\mathrm{Ca}^{2+}$ 探针. 该系列探针的独特之处在于乙二醇基团上所 相连的侧臂, 使探针可以在不影响 BAPTA 基团对 $\mathrm{Ca}^{2+}$ 络合能力的情况下连接到多种功能团上, 实现对 $\mathrm{Ca}^{2+}$ 的定向检测, 检测范围为 $3 \sim 22 \mu \mathrm{mol} / \mathrm{L}$.

\section{$1.2 \mathrm{Cu}^{2+}$ 离子探针}

近年来, 文献[11]报道了多种可用于 $\mathrm{Cu}^{2+}$ 识别和检
测的氧杂葱 Schiff碱系列探针(Schemes 3, 4), 各具特色. 2010 年, $\mathrm{Kim}$ 等 ${ }^{[2]}$ 设计合成的含有 $N$-丁基-1,8-萗二甲酰 亚胺基团的罗丹明基探针 10, 该探针表现出特异的识 别性能, 不仅可以在 $\mathrm{CH}_{3} \mathrm{CN}-\mathrm{HEPES}$ 缓冲体系中通过内 酰胺开环过程选择性的识别 $\mathrm{Cu}^{2+}$ 以形成 $2: 2$ 型配合物, 而且可以通过一个典型的分子内电子转移(ICT) 对 $\mathrm{Zn}^{2+}$ 产生明显的苂光响应而形成 $2: 1$ 型配合物. 2011 年, Miao 等 ${ }^{[13]}$ 报道的探针 6 不仅可以在生理条件下高灵敏 度高选择性的识别 $\mathrm{Cu}^{2+}$, 荧光强度增强超过 40 倍, 而 且可用于 $\mathrm{HeLa}$ 细胞内 $\mathrm{Cu}^{2+}$ 的检测和细胞成像. 2013 年, Wei 等 ${ }^{[14]}$ 设计将糖基团引入到探针 $\mathbf{8}$ 中, 改善了探针的 灵敏度和选择性, 其检测线比世界卫生组织(WHO)规定 的饮用水中 $\mathrm{Cu}^{2+}$ 含量低 200 多倍, 已成功用于饮用水中 $\mathrm{Cu}^{2+}$ 含量的检查, 显现出了其广阔的应用前景.

2012 年, 我们课题组 ${ }^{[15]}$ 也报道了一类罗丹明一肉桂 醛 Schiff 碱型苂光探针 16 (Eq. 3). 研究表明, 该类探针 对 $\mathrm{Cu}^{2+}$ 表现出良好的选择性和较高的灵敏度, 且检测 范围较宽, 探针和 $\mathrm{Cu}^{2+}$ 是形成 $2: 1$ 型配合物. 当向探 针和 $\mathrm{Cu}^{2+}$ 的混合溶液中加入乙二胺时, 溶液颜色变为 无色，荧光消失，表明该系列探针对 $\mathrm{Cu}^{2+}$ 的检测是可逆 的. 细胞成像实验显示该探针可用于活体 HeLa 细胞中 $\mathrm{Cu}^{2+}$ 浓度的检测和分布成像.
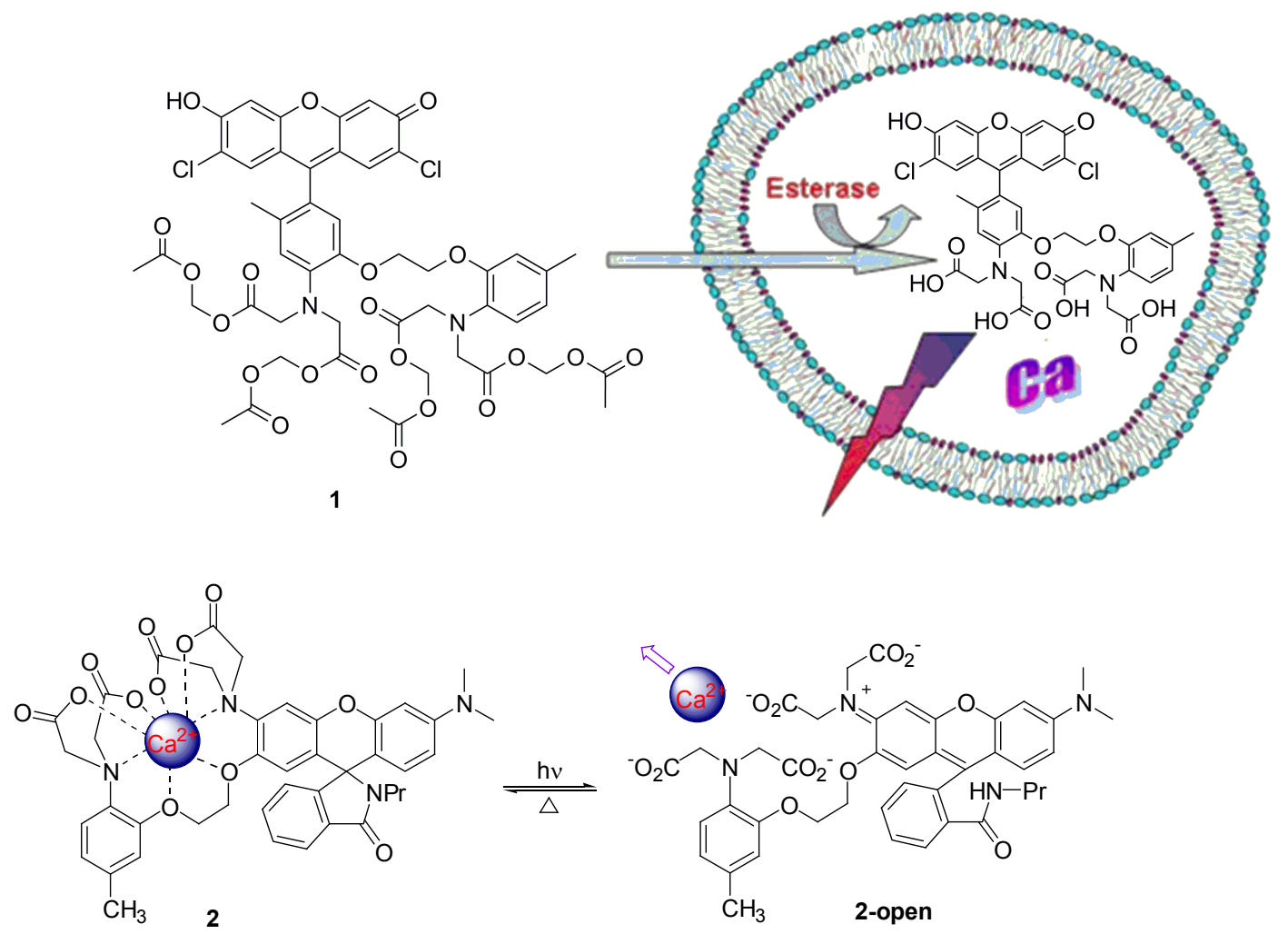


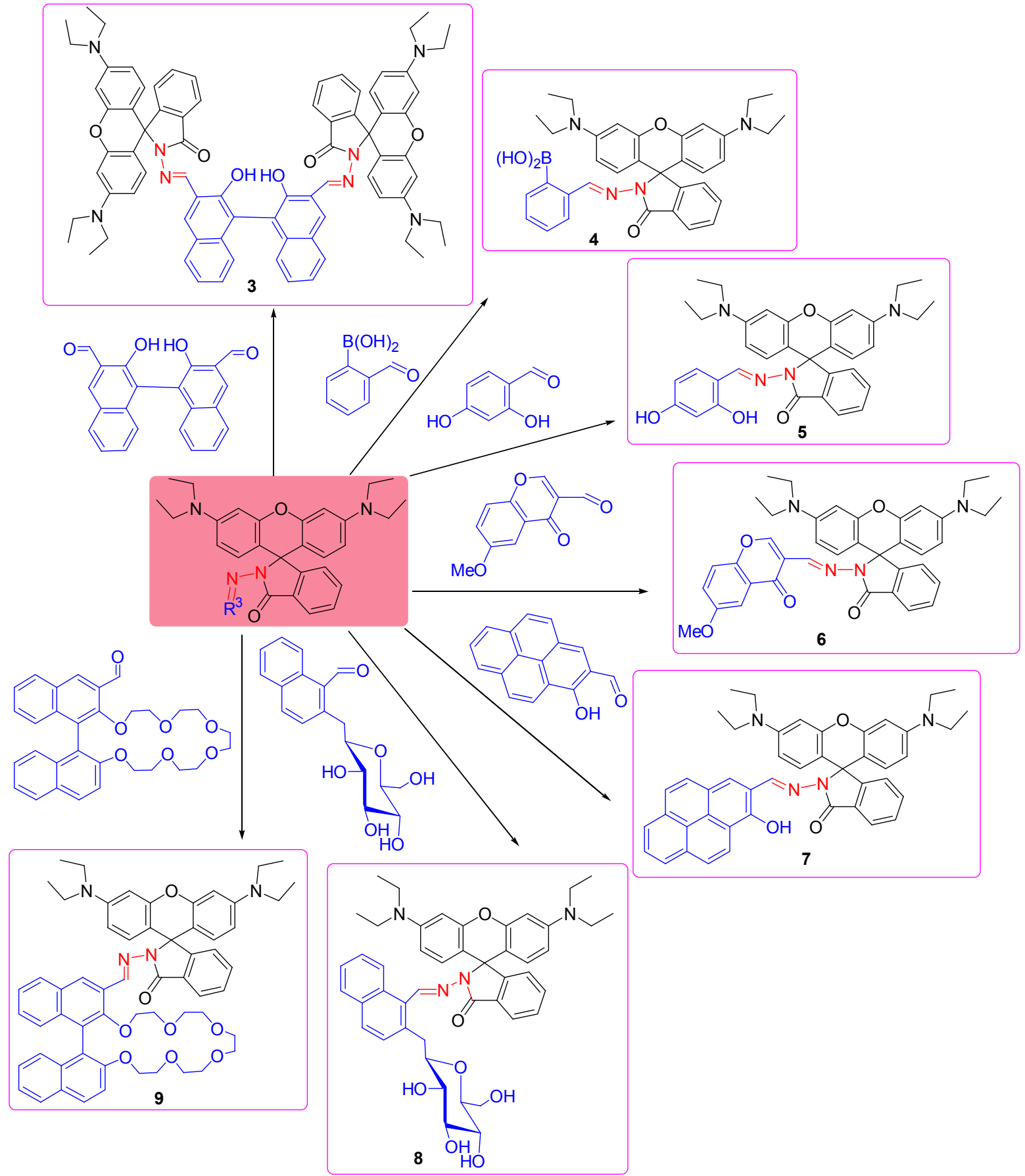

Scheme 3

同年, Lin 等 ${ }^{[16]}$ 设计合成了两种香豆素-罗丹明基新 型 FRET 比率荧光探针 17 (Eq. 4), 该探针是通过环已二 胺将香豆素和罗丹明两种发色团相连, 表现出优异的性 能, 即较大的荧光发射比率和强度、选择性和灵敏度较 高、细胞毒性小和透过性强等. 此类探针综合的性能优 势, 显示了其在生物体系中 $\mathrm{Cu}^{2+}$ 成像识别方面潜在的 应用前景.

在五元螺环隐色体的基础上, 2012 年 Zheng 等 ${ }^{[17]}$ 发
展了一种新型六元螺连隐色体罗丹明型探针，即通过在 罗丹明螺环中嵌入一个 $\mathrm{N}$ 杂原子而获得一种可以在中 性水溶液中检测 $\mathrm{Cu}^{2+}$ 的新型探针 $\mathbf{1 8}(\mathrm{Eq} .5)$. 机理研究 表明, $\mathrm{Cu}^{2+}$ 的加入使氨基硫脲基团发生反应生成异硫氰 酸酯而使氧杂葱重排产生苂光. 六元螺环隐色体设计思 想的引入，克服了此前五元螺环的结构影响及对探针应 用性能拓展所造成的束缚, 为后续性能更优的探针设计 提供了极佳的构建思路. 


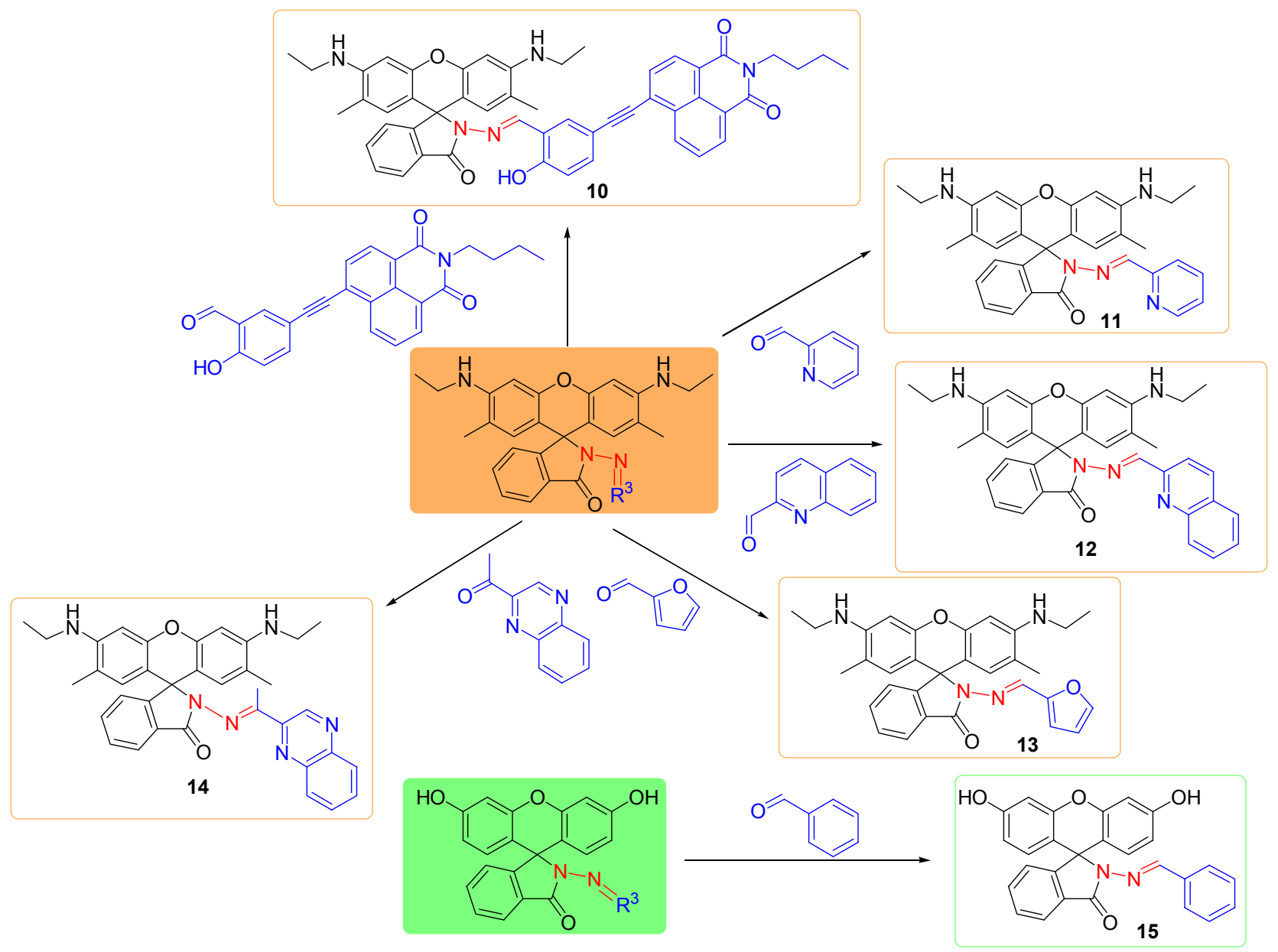

Scheme 4

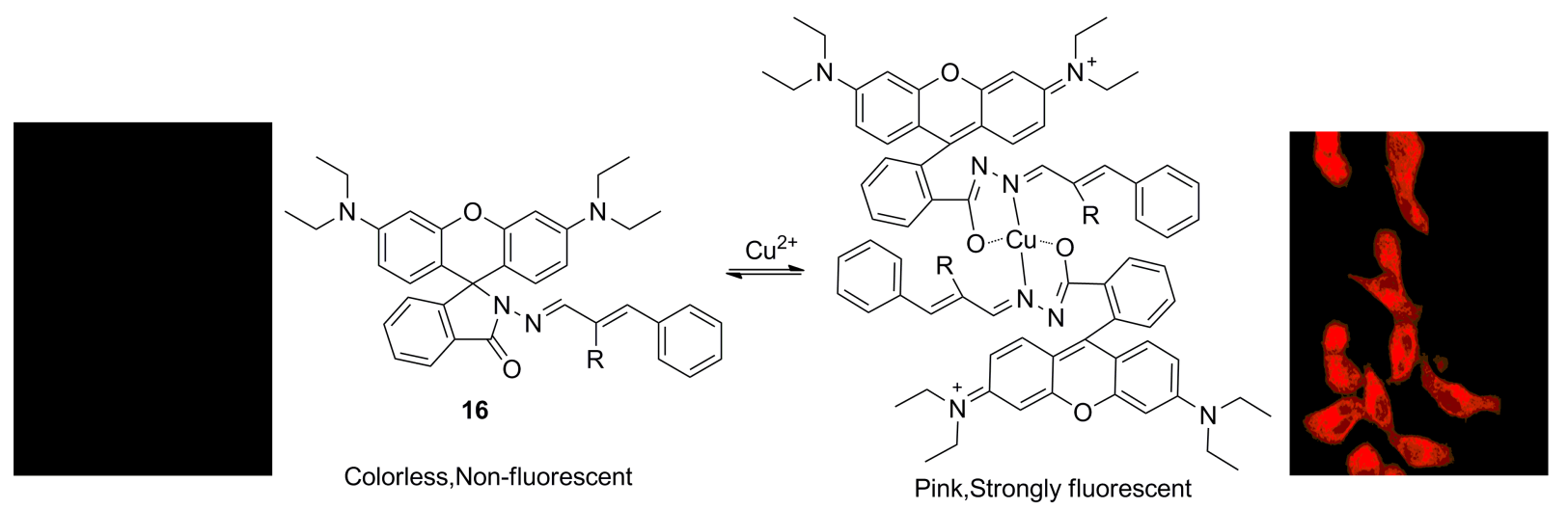

OFF
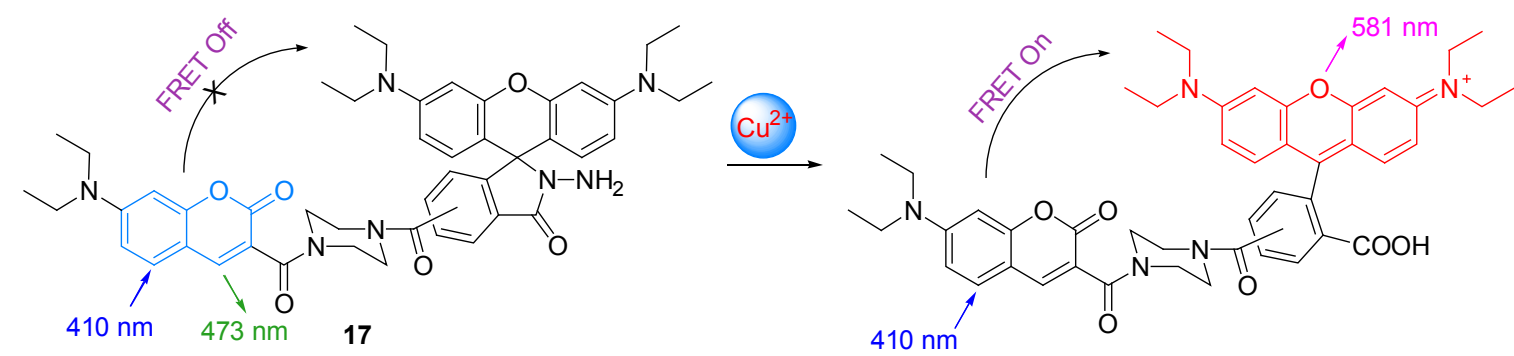


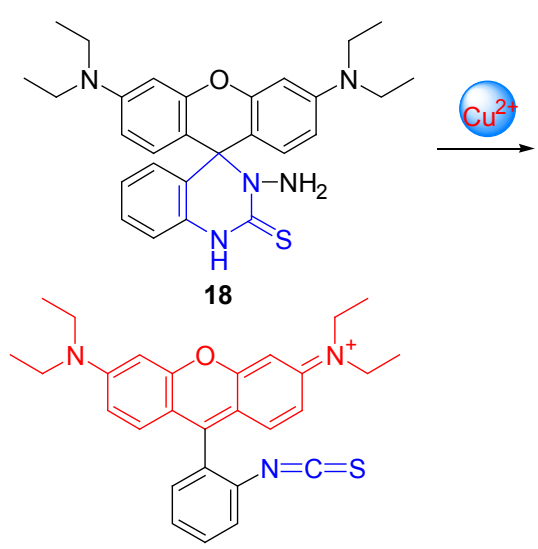

(5)

通过荧光共振能量转移 (FRET) 和跨键能量转移 (TBET)均可获得较大的 Stokes 位移, 相比于 FRET 机理, TBET 并不要求供体和受体之间吸收光谱的重叠, 其应 用性更强. 2013 年, Peng 等 ${ }^{[18]}$ 通过改变萗二甲酰亚胺供 体和罗丹明受体之间的连接基团得到了一种基于 TBET 机理的比率型 $\mathrm{Cu}^{2+}$ 探针 19 (Eq. 6). 该探针目前已成功 应用于活细胞中 $\mathrm{Cu}^{2+}$ 的检测, 其性能表达充分展示了 TBET 型探针的优势, 有助于研究者更好的理解 $\mathrm{Cu}^{2+}$ 在 分子级别生物进程中的重要作用.

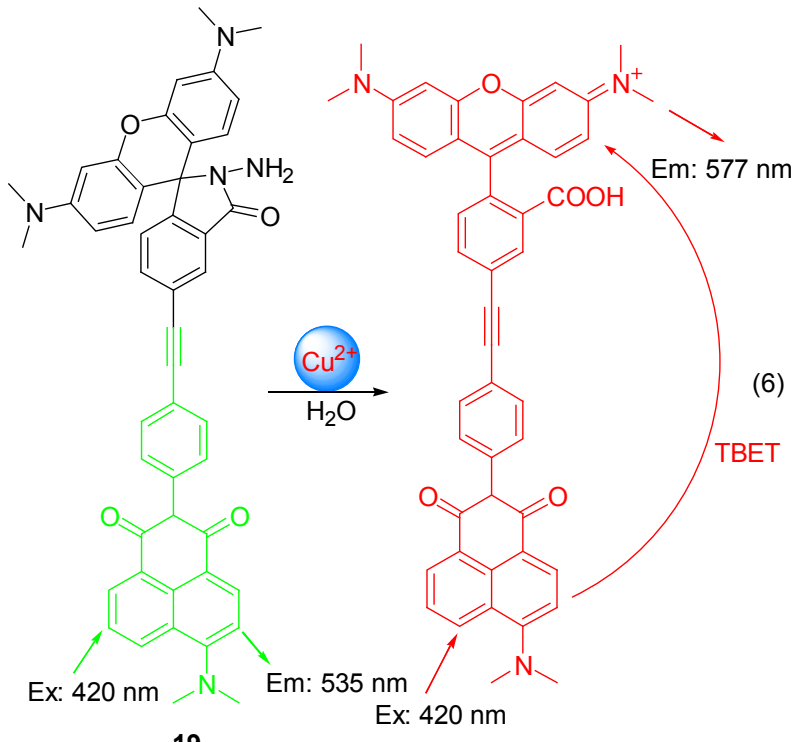

\section{$1.3 \mathrm{Hg}^{2+}$ 离子探针}

与 $\mathrm{Cu}^{2+}$ 相似, 氧杂葱酰肼衍生物也是一类重要的 $\mathrm{Hg}^{2+}$ 探针 ${ }^{[19]} .2007$ 年, Duan 等 ${ }^{[20]}$ 合成了含有吡啶基团的 罗丹明 6G Schiff 碱探针 20 (Scheme 5), 并报道了该探 针与 $\mathrm{Hg}^{2+}$ 形成配合物的单晶结构, 这是首次报道的罗 丹明探针与金属离子所形成的识别络合物单晶结构, 晶 体结构可清晰的显示罗丹明衍生物与 $\mathrm{Hg}^{2+}$ 的识别配位 模式. 同年, Huang 等 ${ }^{[21]}$ 提出将二茂铁基团引入探针分 子，报道了一种可用于 $\mathrm{Hg}^{2+}$ 识别检测的多信号探针 21 .
随后，Duan 等 ${ }^{[22]}$ 也设计合成了包括探针 25 在内的系列 含二茂铁基罗丹明 6G Schiff 碱探针. 研究表明, 该系列 探针的检测限达 $10 \mathrm{nmol} / \mathrm{L} .2011$ 年, Zeng 等 ${ }^{[23]}$ 设计合成 一种对 $\mathrm{Hg}^{2+}$ 表现出较强选择性的新型探针, 并通过 $\mathrm{MCT}$ (碲镉永红外探测)技术对探针生物应用性能进行 了系统的探索，发现此探针的生物应用前景很好.

近年来, 源于 $\mathrm{Hg}^{2+}$ 特殊的结构形式, 应用苂光共振 能量转移(FRET) 原理设计合成新型 $\mathrm{Hg}^{2+}$ 探针逐渐成为 新的发展趋势. 2010 年, Kim 等 ${ }^{[24]}$ 将两个萠基团连接到 1,3-交替的杯 [4]芳烃上，进而将其与罗丹明荧光团桥连 得到一种可选择性识别 $\mathrm{Hg}^{2+}$ 的探针 32 (Eq. 7). 相比于 分子间 $\pi-\pi$ 相互作用, 分子内 $\pi-\pi$ 相互作用力形成的 FRET 效率更高. 但由于该探针检测需要在纯有机溶剂 条件下，这限制了其进一步的生物苂光应用前景. 随后， Zeng 等 ${ }^{[25]}$ 设计报道了含有 $\beta$-环糊精基团的探针 33 (Eq. 8 ), $\beta$-环糊精基团不仅可以作为连接能力供体和受体之 间的桥梁，还可大大提高探针的水溶性，苂光显微成像 实验表明这种 FRET 识别体系可成功用于活细胞中 $\mathrm{Hg}^{2+}$ 检测. 2011 年, $\mathrm{Wu}$ 等 ${ }^{[26]}$ 则巧妙的将甲基丙烯酸甲 酯和丙烯酸通过乳液聚合得纳米粒子, 然后将硝基苯并 呋咱(NBD)引入到纳米粒子的核心，再将罗丹明基团嫁 接到纳米粒子表面得到 FRET 基比色探针 34 (Eq. 9), 在 中性体系下对 $\mathrm{Hg}^{2+}$ 的检测限可达 $100 \mathrm{nmol} / \mathrm{L}$.

2011 年, Kumar 等 ${ }^{[27]}$ 富有创意的将荎二甲酰亚胺和 罗丹明相串接，首例报道了一种 TBET 原理下的 $\mathrm{Hg}^{2+}$ 探 针 35 (Eq. 10). 2012 年, 该研究组 ${ }^{[28]}$ 又报道了 TBET 型 罗丹明-并五苯二酮基探针 36 (Eq. 11), 此探针对 $\mathrm{Hg}^{2+}$ 表现出良好的选择性且不受共存金属离子的干扰，应用 于活细胞中 $\mathrm{Hg}^{2+}$ 的检测. 随后, Zhang 等 ${ }^{[29]}$ 设计的比率 型 $\mathrm{Hg}^{2+}$ 探针 37 (Eq. 12), 通过将 TBET 思路和双转换设 计相结合, 得到了两个独立的具有较高能量转移效率的 发射带, 对 TBET 型探针的发展提供了新的方向. 该探 针具有较高的灵敏度, 不但可成功应用于活细胞检测, 尤其重要的是可应用于环境水质样品中 $\mathrm{Hg}^{2+}$ 的检测.

近年来, 随着材料化学领域的不断发展, 将有机一 无机混合材料应用于离子识别引起了研究工作者的关 注. 这些无机受体材料, 例如 $\mathrm{SiO}_{2}, \mathrm{Al}_{2} \mathrm{O}_{3}$ 和 $\mathrm{TiO}_{2}$, 在固液多相检测体系中具有独特的优势. 2012 年, Yang 等 ${ }^{[30]}$ 通过自组装和静电纺丝技术得到可作为 $\mathrm{Hg}^{2+}$ 探针的纳 米复合材料纤维 38 (Scheme 6). 由于静电纺丝的比表面 积较大，容易进行表面修饰，从而增大亲水性. 通过自 组装而使静电纺丝和探针分子结合起来增加了探针的 灵敏度，从而实现快速检测。该纳米薄膜可高灵敏度高 选择性的检测 $\mathrm{Hg}^{2+}$ ，检测限为 $0.6 \mu \mathrm{mol} / \mathrm{L}$. 该项技术的 应用为研发可在水溶液中对金属离子进行检测的高性 


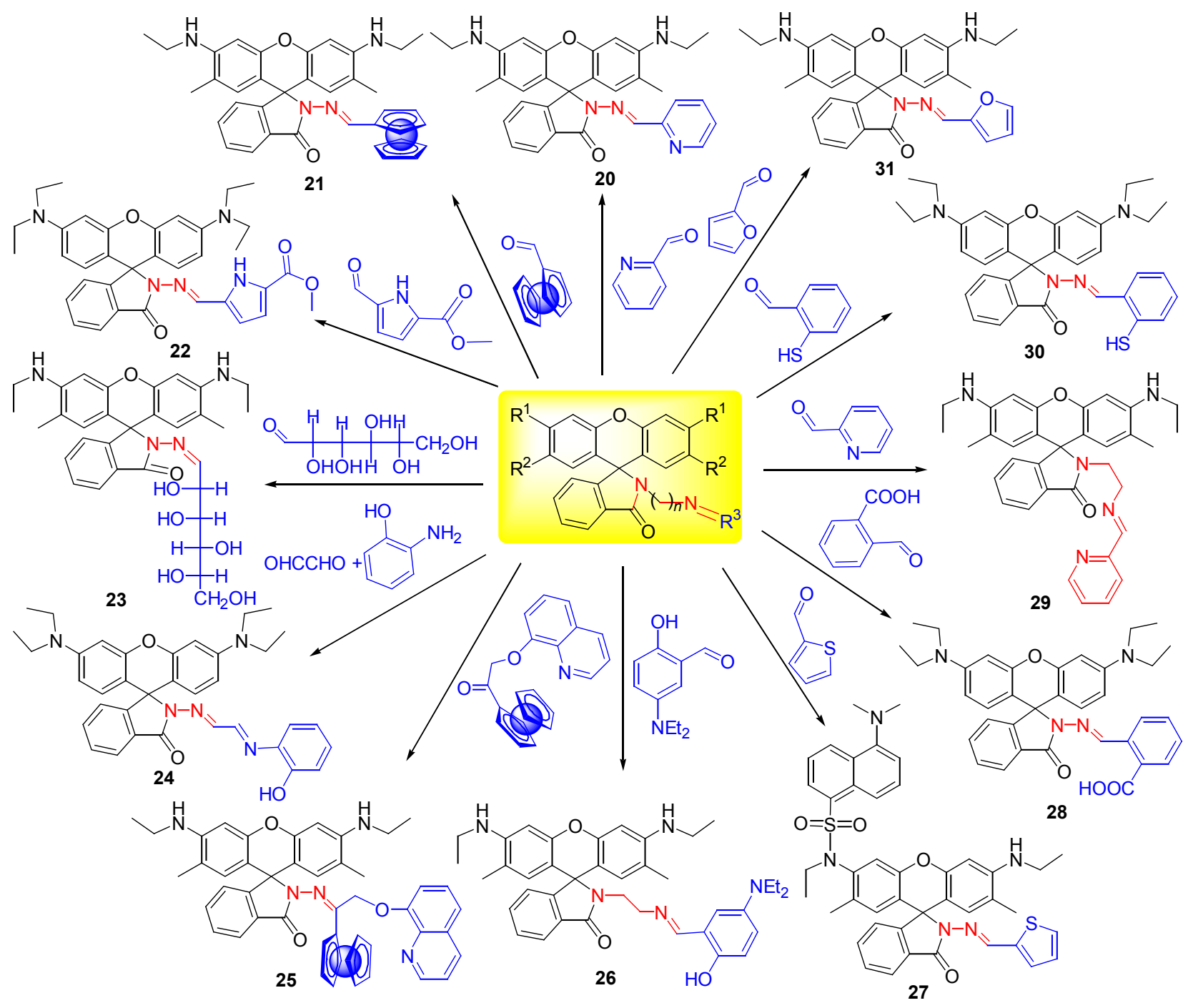

Scheme 5
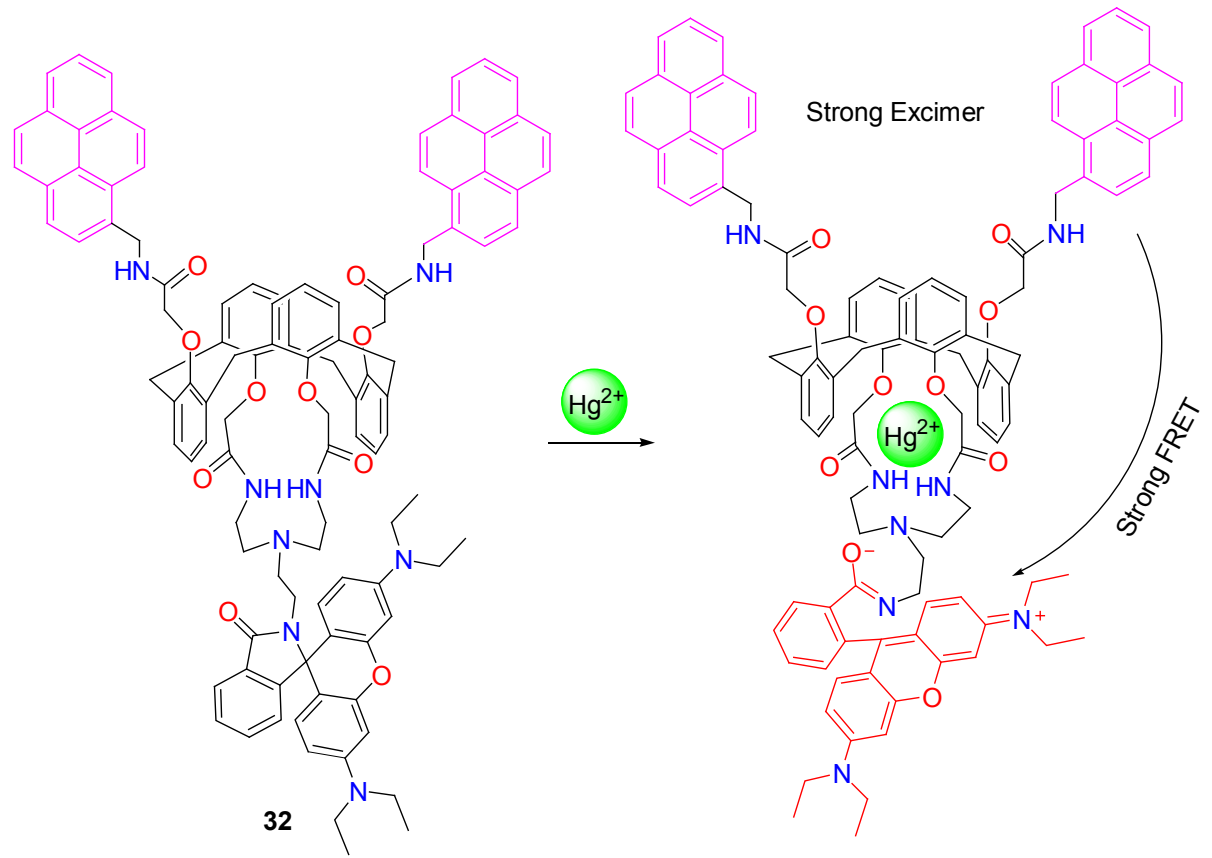


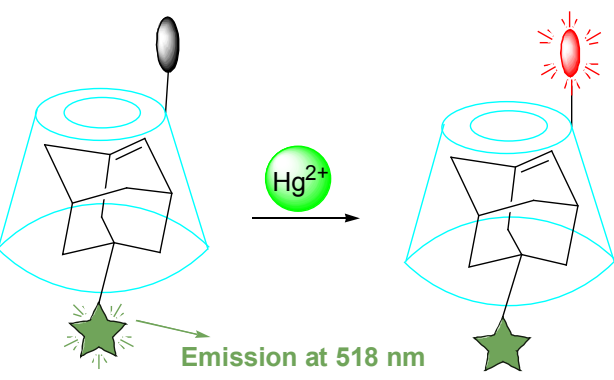

$\xi={ }_{S}$<smiles>CCN(CC)c1ccc2c(c1)Oc1cc(N(CC)CC)ccc1C21c2ccccc2C(=O)N1CCNC(=S)NCCNC</smiles>

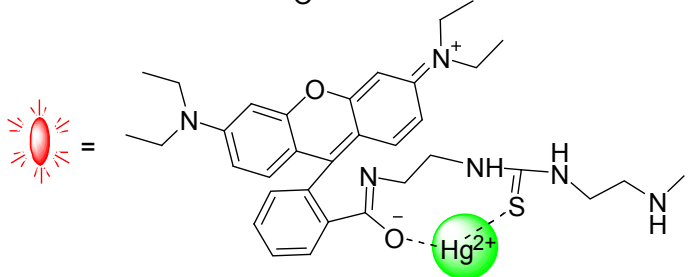
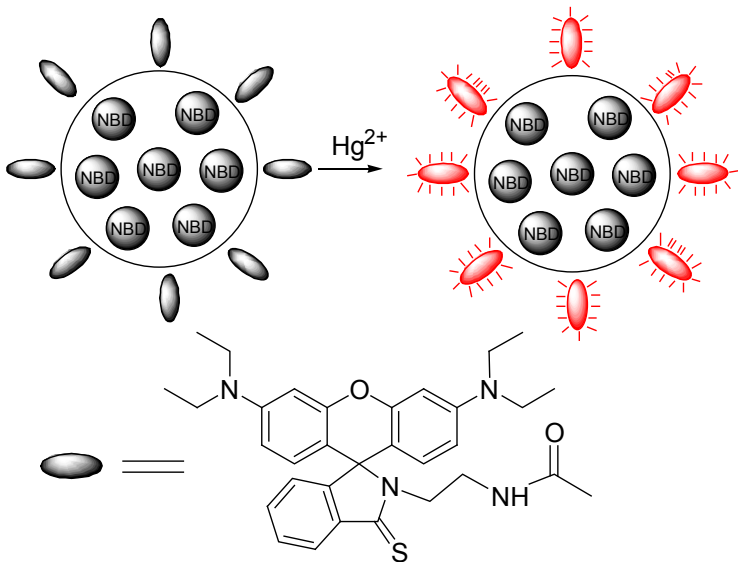

34
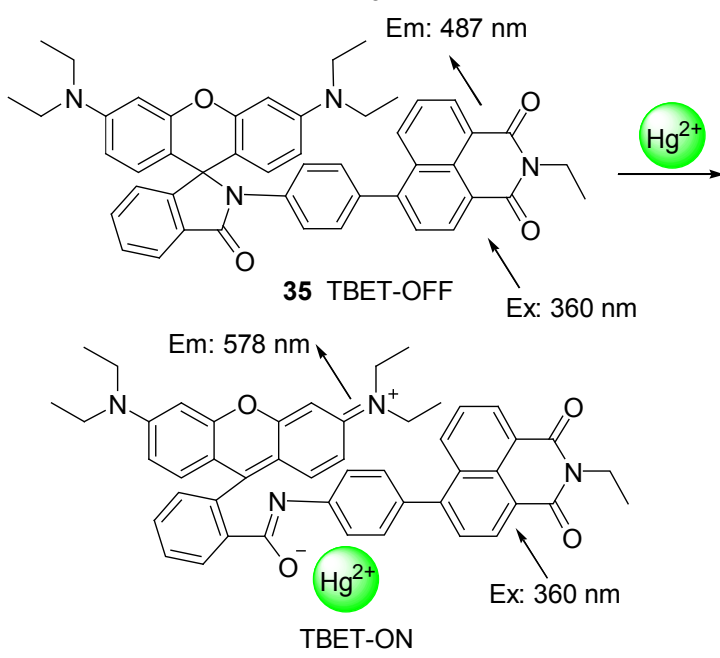

(8)<smiles>CCN(CC)c1ccc(-c2cc3cc4c(cc3cc2-c2ccc(N3C(=O)c5ccccc5C35c3ccc(N(CC)CC)cc3Oc3cc(N(CC)CC)ccc35)cc2)C(=O)c2ccccc2C42c3ccc(N(CC)CC)cc3Oc3cc(N(CC)CC)ccc32)cc1</smiles>

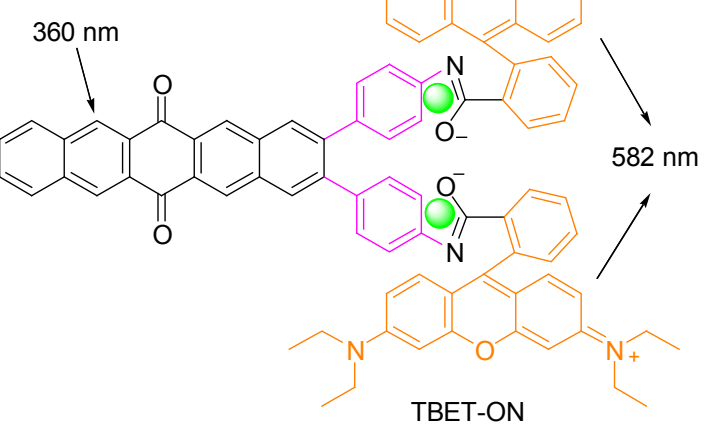

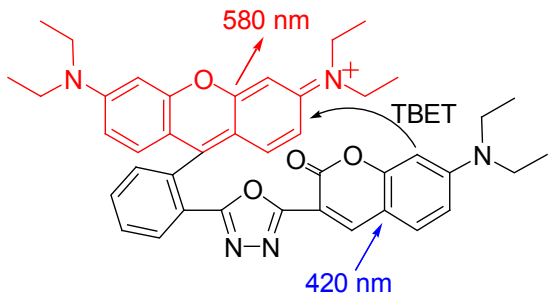

能检测材料提供了一种良好的选择，也为分子探针的发 展开辟了新的领域.

2013 年, $Y e$ 等 $^{[31]}$ 设计合成了一种可在苂光和肉眼 条件下识别检测 $\mathrm{Hg}^{2+}$ 的罗丹明基荧光探针 38 (Scheme 7). 该探针分子中含有亲水的磷酸酯基团，可在较宽的 $\mathrm{pH}$ 范围的水溶液中对 $\mathrm{Hg}^{2+}$ 表现出高灵敏度和高选择性 的响应，检测线可达 $0.6 \mu \mathrm{mol} / \mathrm{L}$. 该探针的检测机理可 理解为: 在 $\mathrm{Hg}^{2+}$ 诱导下, 探针发生内酰胺开环反应, 脱 去 $\mathrm{HgS}$ 形成咪唑啉环, 然后水解得到最终的开环产物发 生荧光和颜色变化. 此外, 在活体细胞 MGC803 中的荧 光成像实验表明，该探针具有进一步实现检测活细胞中 

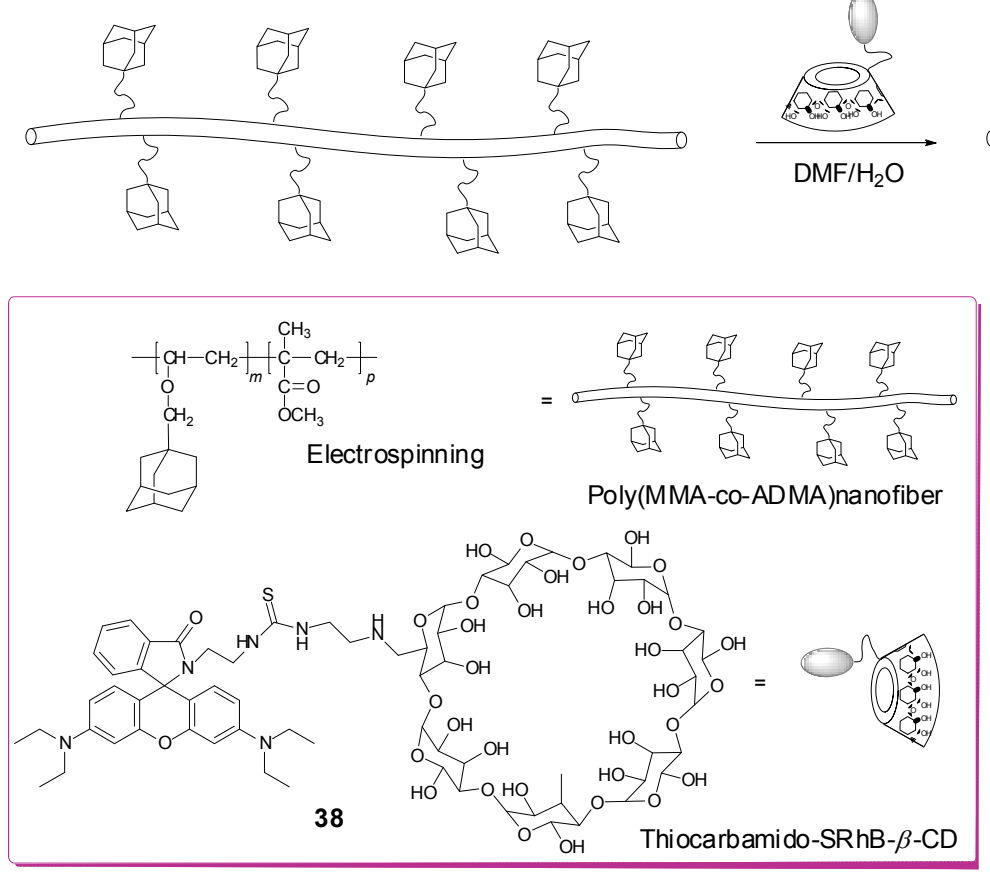

Scheme 6

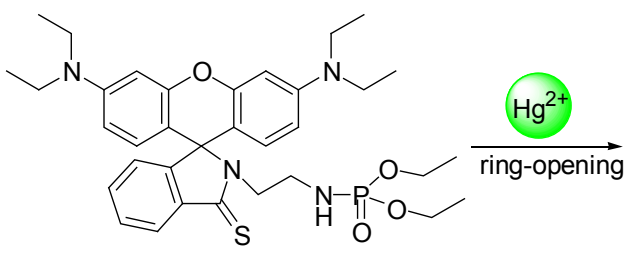

39

Colorless, Non-fluorescent

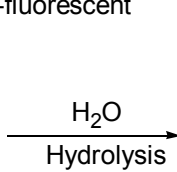

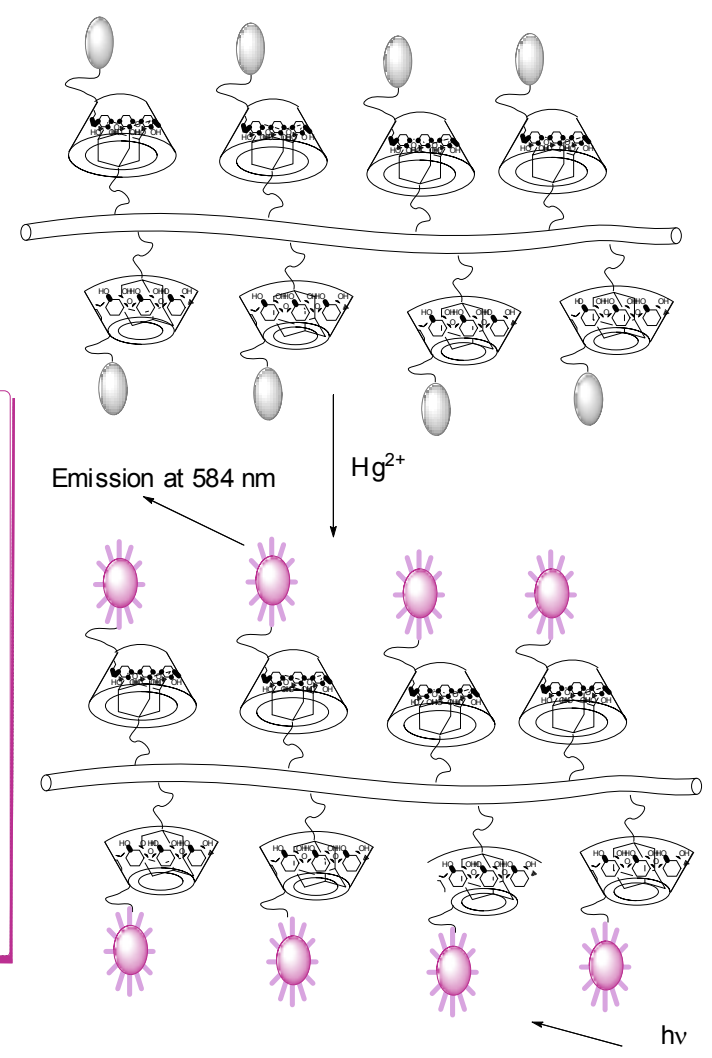

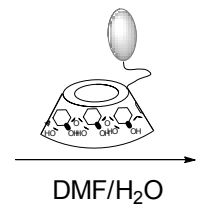$$
\text { (n) }
$$
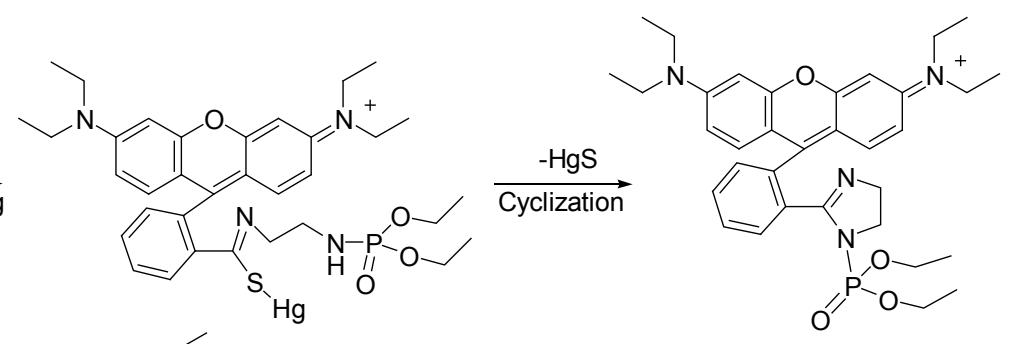

Pink, Strong fluorescent

Pink, Strong fluorescent

Scheme 7

$\mathrm{Hg}^{2+}$ 含量的潜在能力.

2013 年, 我们课题组 ${ }^{[32]}$ 设计合成报道了一种含有 六元螺内酰肼结构的 $\mathrm{Hg}^{2+}$ 探针 40 (Eq.13). 研究表明, 苯并噻唑基团引入不仅可实现六元螺环的形成, 同时也 为 $\mathrm{Hg}^{2+}$ 的识别提供了更多的柔性结合位点, 优化了探 针的菼光强度和光稳定性. 当向探针 40 的溶液中加入 $\mathrm{Hg}^{2+}$ 后, 其荧光强度增强超过 1000 倍, 且能长时间保持 不减退或淬灭. 该探针已成功的用于活细胞和细菌EPS-矿物聚集体中 $\mathrm{Hg}^{2+}$ 的检测, 这也是首次报道的可 用于细菌-EPS-矿物聚集体 $\mathrm{Hg}^{2+}$ 检测的氧杂蒽荧光探
针.

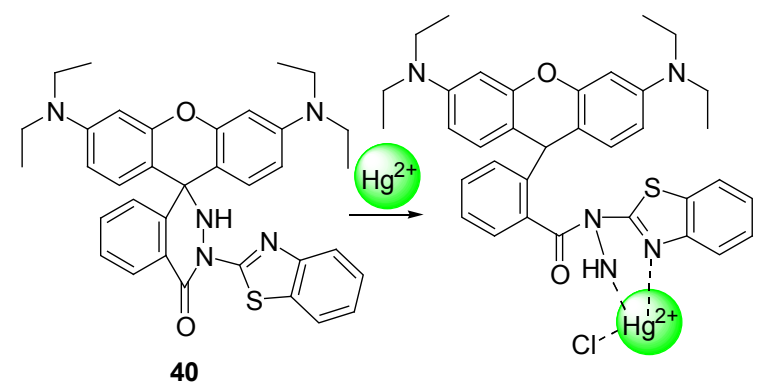




\section{$1.4 \mathrm{Zn}^{2+}$ 离子探针}

2010 年, Zhang 等 ${ }^{[33]}$ 基于 FRET 原理, 报道了一种 可在水溶液中检测 $\mathrm{Zn}^{2+}$ 的罗丹明衍生物 41 (Eq. 14), 检 测限为 $0.4 \mathrm{nmol} / \mathrm{L}$. 该探针分子中含有两个功能团, 一 个是罗丹明硫酰肼, 另一个是荧光素. 在此 FRET 系统 中, 菼光素作为供体而罗丹明作为受体, 罗丹明中的 $\mathrm{S}$ 原子、 $\mathrm{N}$ 原子和荧光素中的 $\mathrm{O}$ 原子共同作用, 选择性的 与 $\mathrm{Zn}^{2+}$ 配位诱导内酰胺开环而使罗丹明基团产生苂光. 探针 41 对 $\mathrm{Zn}^{2+}$ 表现出较强的选择性, 即使是和 $\mathrm{Zn}^{2+}$ 竞 争作用很强的 $\mathrm{Cd}^{2+}$ 也对识别没有干扰. 由于探针 41 供 体和受体的发射波长之间相差较大, 并且具有检测分辨 率高、检测时间短和检测过程可逆等优点, 因此具有应 用于细胞成像方面的潜在价值.

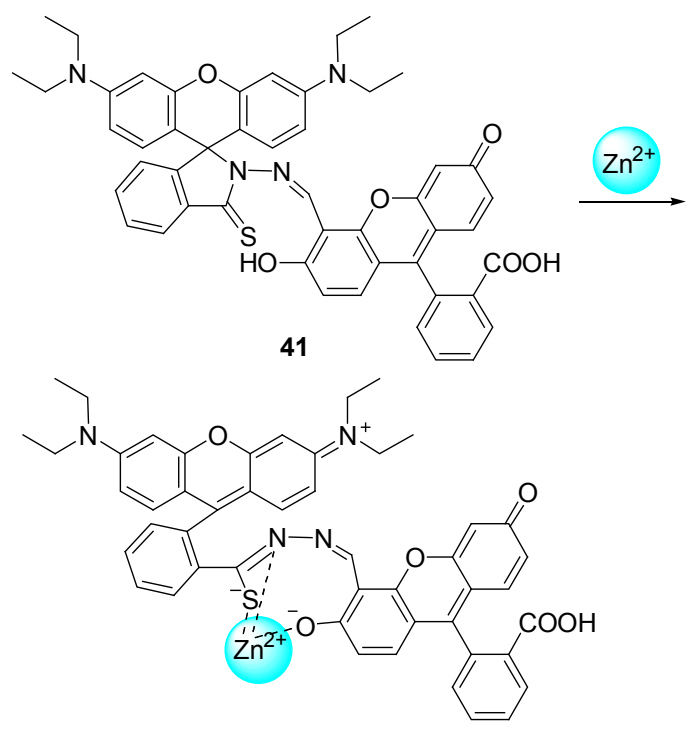

2011 年, Taki 等 ${ }^{[34]}$ 设计合成了一种连有胆固醇基团 的菼光素基 $\mathrm{Zn}^{2+}$ 探针 42 (Scheme 8). 胆固醇基团的加入 使该探针可穿透细胞膜, 检测细胞中 $\mathrm{Zn}^{2+}$ 的浓度及其 局部分布信息. 与遗传编码技术不同，通过变换不同的 发光团, 可以实现对同一单个细胞的不同区域 $\mathrm{Zn}^{2+}$ 的 检测和细胞成像, 使用这种成像技术将有助于研究工作 者更好的了解细胞器之间 $\mathrm{Zn}^{2+}$ 的转移路径.

2011 年, Qian 等 ${ }^{[35]}$ 也报道了一种多用的罗丹明基三 功能荧光探针 43 (Eq. 15). 该探针不仅可以在 DMSO$\mathrm{H}_{2} \mathrm{O}$ 溶液中高灵敏度高选择性的识别 $\mathrm{Cu}^{2+}$, 并可以通过 肉眼观察到从无色到紫色的颜色变化, 还可以在 EtOH- $\mathrm{H}_{2} \mathrm{O}$ 溶液中选择性的识别 $\mathrm{Zn}^{2+}$, 出现 OFF-ON 型 荧光发射并伴随溶液颜色从无色到紫色的变化. 这是首 次报道的可在不同的水溶液中分别对 $\mathrm{Cu}^{2+}$ 和 $\mathrm{Zn}^{2+}$ 产生 响应的苂光探针. $\left[43_{2} \mathrm{Zn}^{2+}\right.$ ]配合物是有色有荧光的物质, 它可以对 His/Cys 产生双重响应. 此外, 苂光共聚焦成 像实验表明 43 可用于活的 HeLa 细胞中 $\mathrm{Zn}^{2+}$ 检测和细 胞成像.

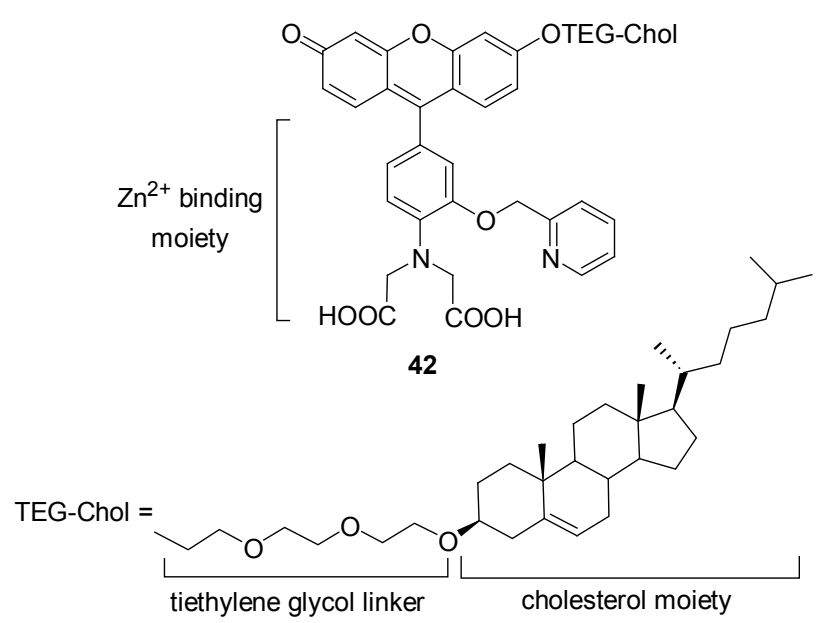

Scheme 8

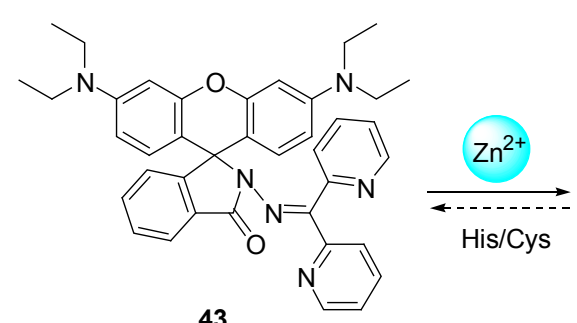

43

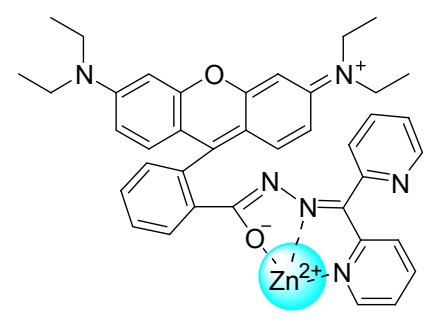

2012 年, Zhu 等 ${ }^{[36]}$ 设计合成了一种通过分子内电子 转移(ICT)而发光的罗丹明衍生物 44 (Scheme 9). 该探 针可通过可见光激发, 在乙腈中随着 $\mathrm{Zn}^{2+}$ 浓度的升高 而发生连续红移. $\mathrm{Zn}^{2+}$ 首先结合到双吡啶基团上, 稳定 ICT 的激发态使颜色从蓝色变为绿色, 而高浓度的 $\mathrm{Zn}^{2+}$ 诱导罗丹明内酰胺开环，探针发生分子内 FRET 而使颜 色从绿色变为橙色. 该工作为此后设计新型探针提供了 良好的思路，即在不同浓度梯度下，探针分子发射波长 产生位移而使其产生不同的颜色变化，具有广阔的应用 前景.

\section{$1.5 \mathrm{~Pb}^{2+}$ 离子探针}

2011 年, $\mathrm{Kim}$ 等 ${ }^{[37]}$ 设计合成了两种罗丹明衍生物 $\mathbf{4 5}$ 和 46 并将其组装到玻璃表面得到了可在 $\mathrm{CH}_{3} \mathrm{CN}$ 溶液中 选择性检测 $\mathrm{Pb}^{2+}$ 的荧光探针 (Scheme 10). 这两种探针 的响应方式均是在 $\mathrm{Pb}^{2+}$ 诱导下罗丹明内酰胺开环产生 的信号变换, 且这种转换是完全可逆的, 可重复使用. 该类探针的缺点是水溶性较差，限制其进一步的应用推 


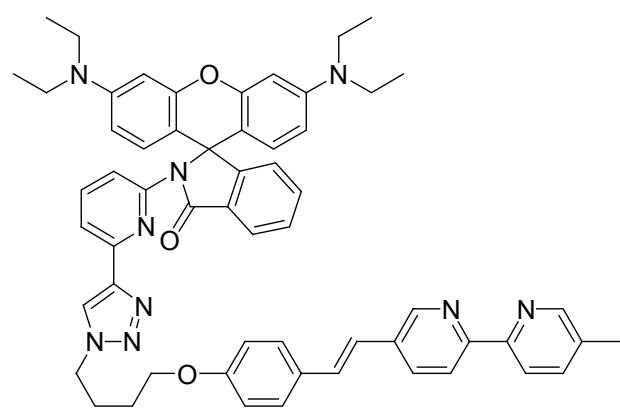

44
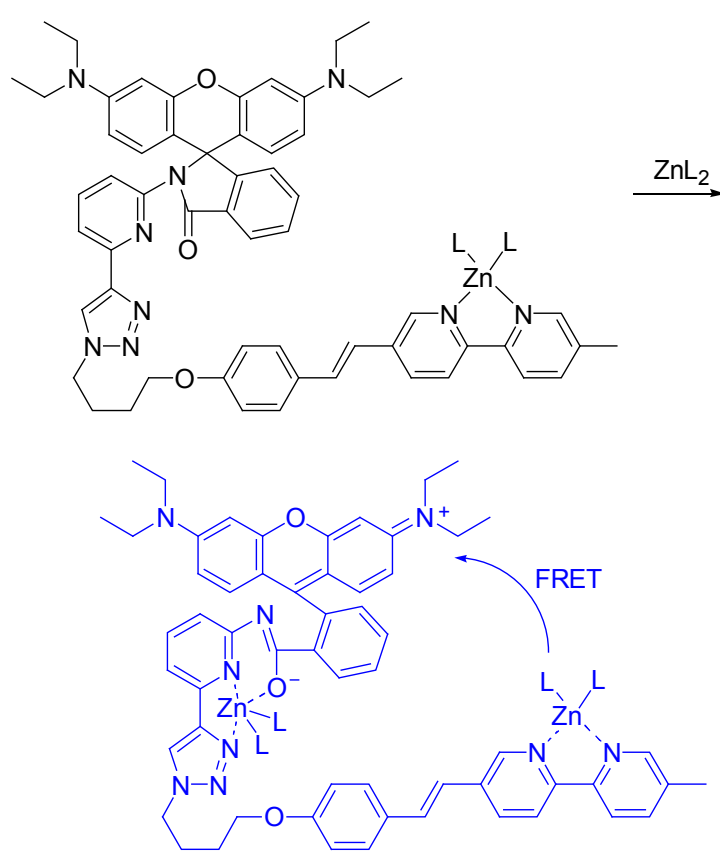

Scheme 9

广，尤其是在水质样品中金属离子的选择性荧光响应较 差.

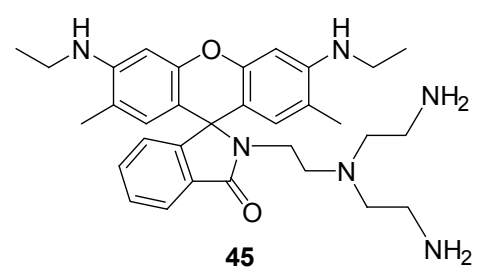<smiles>CCNc1cc2c(cc1C)C1(O2)c2ccccc2C(=O)N(CCNCCN)c2cc(NCC)c(C)cc21</smiles>

Scheme 10

2012 年, Tyagi 等 ${ }^{[38]}$ 设计将罗丹明 $6 \mathrm{G}$ 连接到 $\mathrm{Ag}$ 纳
米棒表面, 得到了一种可在水溶液中对 $\mathrm{Pb}^{2+}$ 产生高灵敏 度高选择性响应的探针 47 (Eq. 16), 这也是首例文献报 道的应用银纳米棒探针检测 $\mathrm{Pb}^{2+}$ 的研究方法. 当向该体 系的溶液中加入 $\mathrm{Pb}^{2+}$ 时, 可在 $3 \mathrm{~min}$ 内快速产生响应并 可用肉眼观察到颜色变化，且不受其它共存离子的干 扰，检测限为 $28 \mathrm{nmol} / \mathrm{L}$. 该方法具有简单、快速、成本 低廉的优点, 应用推广前景开阔。
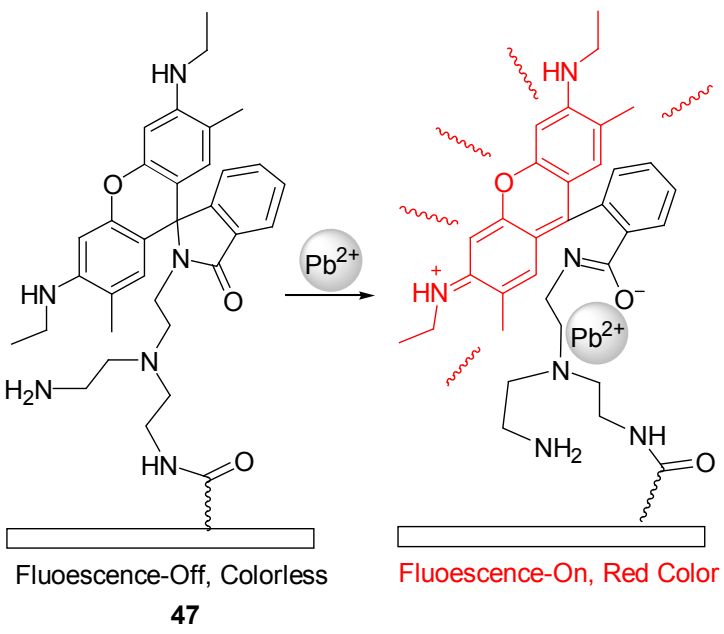

\section{$1.6 \mathrm{Fe}^{3+}$ 离子探针}

2010 年以来, $\mathrm{Fe}^{3+}$ 苂光探针研发突飞猛进, 而 Schiff 碱类型探针则又一次引领了 $\mathrm{Fe}^{3+}$ 探针的发展潮流 (Scheme 11). 在构筑 Schiff 碱官能团过程中, 喹啉基团 受到了特别的关注. 2011 年以来, $\mathrm{Zhu}^{[39]}$, Thennarasu ${ }^{[40]}$ 和 Zeng 等 ${ }^{[41]}$ 均报道了含有喹啉官能团的罗丹明基 $\mathrm{Fe}^{3+}$ 探针. 其中 Zeng 等报道的探针 $\mathbf{5 1}$ 性能较为优异, 可在 高浓度细胞内阳离子和环境中有毒金属离子的存在下, 高灵敏度高选择性的检测 $\mathrm{Fe}^{3+}$, 且形成 $1: 1$ 型配合物, 检测范围为 $0 \sim 100 \mu \mathrm{mol} / \mathrm{L}$. 相比之下, Ramakrishna ${ }^{[42]}$ 和 Kumar 等 ${ }^{[43]}$ 设计的双罗丹明基探针 54, 55 和 56, 由 于使用双电子激发, 有效的降低了吸收干扰并且增加了 双电子的横截面, 因此苂光强度更强, 应用性能看好.

2010 年, Zeng 等 ${ }^{[4]}$ 巧妙地合成了一种含有两个罗 丹明基团连接着 1,3-交替的硫杂杯 [4]芳烃的荧光探针 57 (Eq. 17), 该探针可以在 $\mathrm{EtOH}-\mathrm{H}_{2} \mathrm{O}$ 溶液中选择性的 检测 $\mathrm{Fe}^{3+}$ 和 $\mathrm{Cr}^{3+}$. 研究表明该探针和金属离子是 $1: 1$ 配位的. IR 吸收光谱显示当加入 20 倍金属离子时，酰胺 羰基的吸收峰从 $1686 \mathrm{~cm}^{-1}$ 降低到 $1590 \mathrm{~cm}^{-1}$, 而 ${ }^{1} \mathrm{H}$ $\mathrm{NMR}$ 光谱也表明羰基 $\mathrm{O}$ 原子和 $\mathrm{Fe}^{3+}$ 或 $\mathrm{Cr}^{3+}$ 配位. 因此, 推测该探针的识别机理是金属离子的诱导使内酰胺开 环, 进而出现苂光增强现象并伴随着颜色从无色到紫色 的变化. 

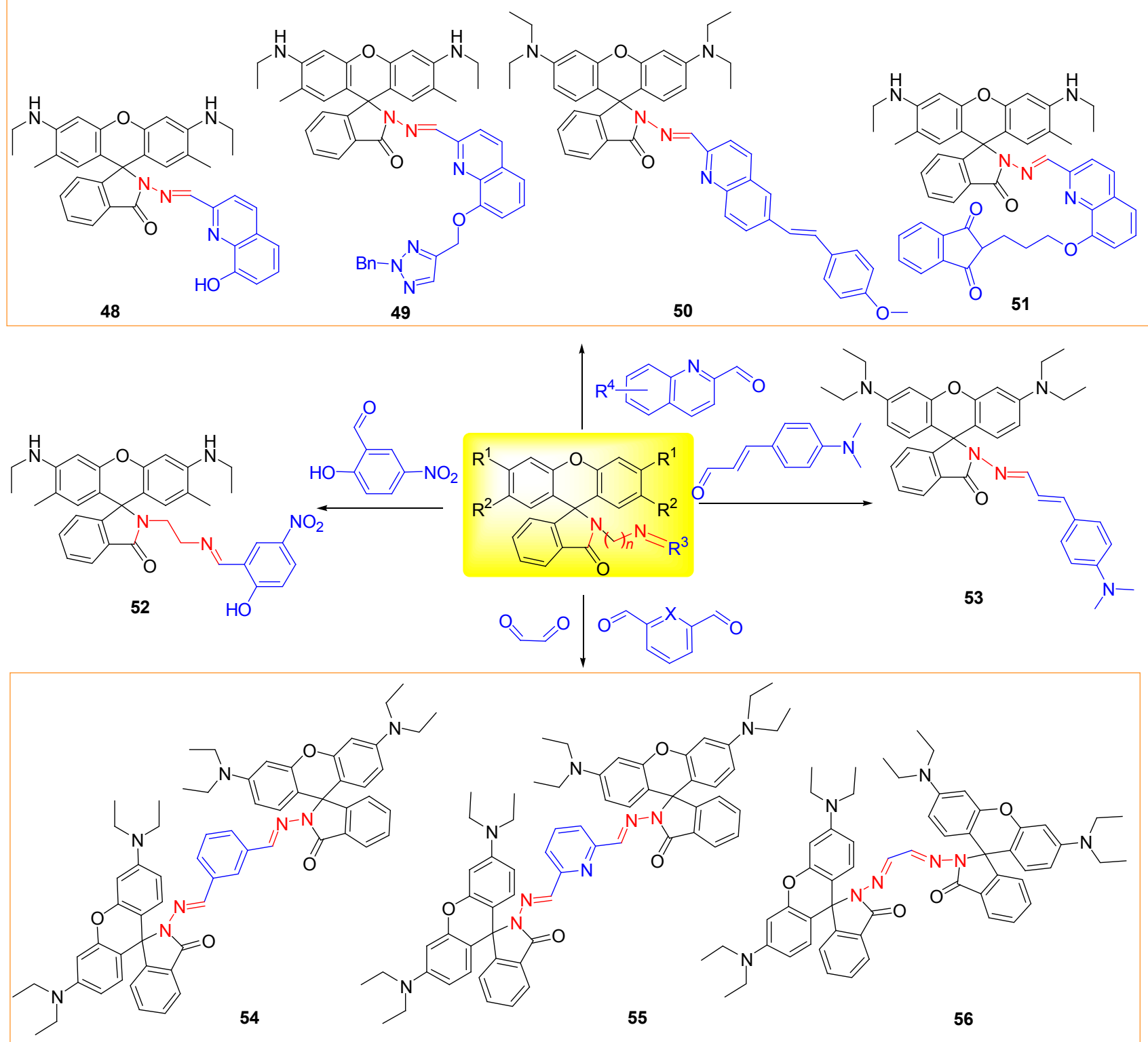

Scheme 11

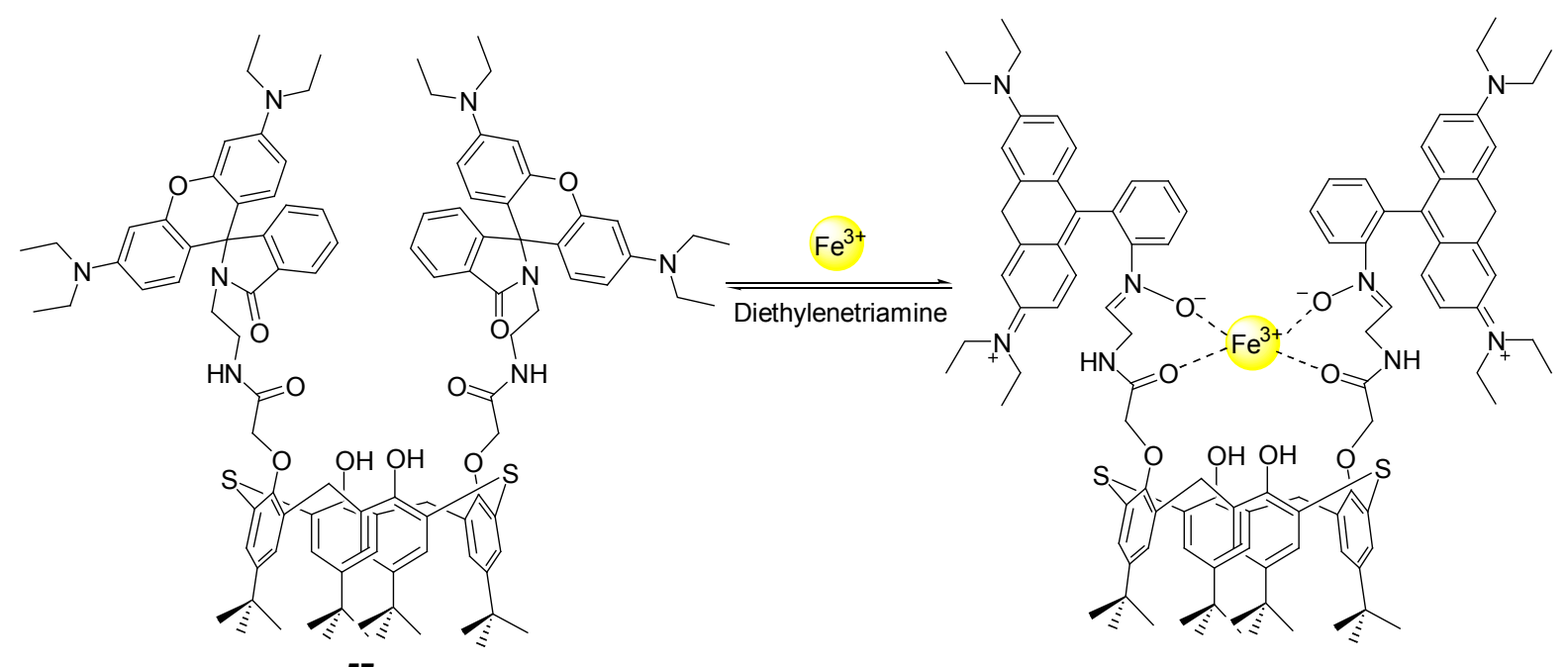


$\mathrm{Ma}$ 等 $^{[45]}$ 使用聚环氧乙烷- $b$-聚苯乙烯作为基本骨架 构建可在水溶液中检测 $\mathrm{Fe}^{3+}$ 的 FRET 型比色探针 $\mathbf{5 8}$ (Scheme 12). 该探针分子中, 疏水的苂光染料 NBD 位 于胶团的核心位置, 作为 FRET 体系的能量供体, 而另 一种内酰胺罗丹明衍生物 $(\mathrm{SRhB}-\mathrm{OH})$ 吸附在聚合物表 面作为能量受体. 当体系中加入 $\mathrm{Fe}^{3+}$ 时, 即发生 $\mathrm{Fe}^{3+}$ 诱 导的内酰胺开环反应, 并通过 FRET 体系使探针产生苂 光, 检测限为 $1.0 \mu \mathrm{mol} / \mathrm{L}$. 通过变换 FRET 体系的能量供 体和受体, 则可以调控探针性能用于检测不同的金属离 子.

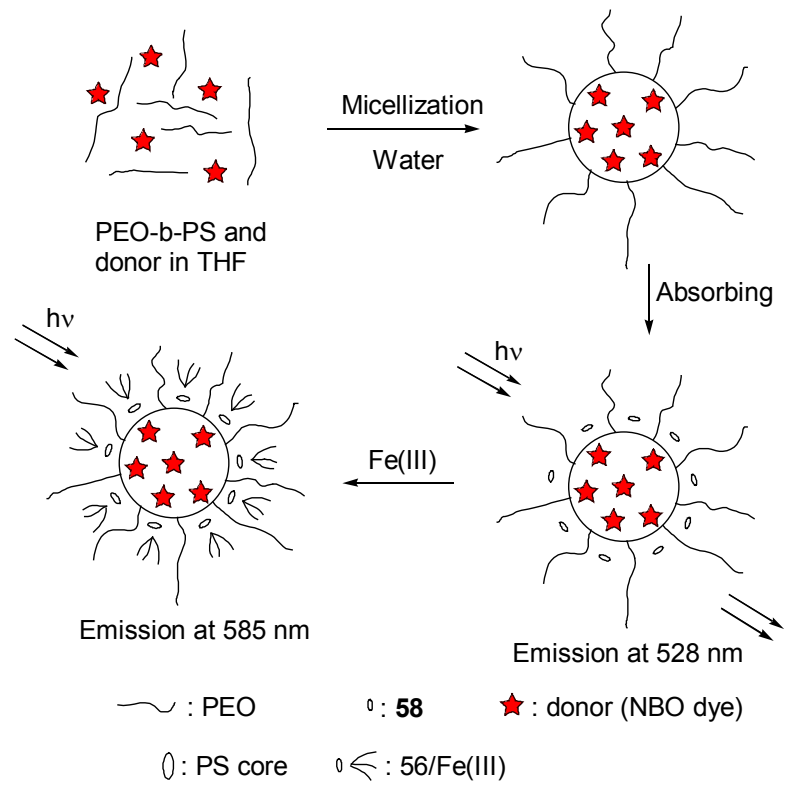

Scheme 12
$\mathrm{Wu}$ 等 ${ }^{[46]}$ 报道了一种聚合物探针 59 (Eq. 18), 该探 针可像试纸一样很容易的检测水溶液中的 $\mathrm{Fe}^{3+}$, 检测限 为 $1.0 \mu \mathrm{mol} / \mathrm{L}$. 在该探针分子中, 通过低毒性且生物相 容性较好的聚乙烯醇(PVA)将含有内酰胺螺环的罗丹明 衍生物和丹磺酰基团相连接，形成一种基于 FRET 检测 原理的荧光探针. 在该体系中，丹磺酰基团为能量供体， 而罗丹明基才则是能量受体. 当向该探针的溶液中加入 $\mathrm{Fe}^{3+}$ 时，罗丹明内酰胺开环，探针分别在 489(丹磺酰)和 594 (罗丹明 B) nm 处出现两个苂光发射, 其苂光强度随 $\mathrm{Fe}^{3+}$ 浓度的变化而呈比率变化.

为了克服有机苂光探针分子普遍水溶性较差, 所导 致的在生物体系中探针对金属离子检测灵敏度低的弊 端, Yang 等 ${ }^{[47]}$ 提出使用纳米粒子来增强探针的水溶性, 即通过聚乙烯醇(PEG)将罗丹明 $6 \mathrm{G}$ 乙二胺探针 60 和磁 性的 $\mathrm{Fe}_{3} \mathrm{O}_{4}$ 纳米粒子相连接. 该探针设计思路不仅可以 大大提高探针的水溶性, 还可通过磁性将 $\mathrm{Fe}^{3+}$ 从有机溶 剂中分离, 以增强探针对 $\mathrm{Fe}^{3+}$ 的选择性, 检测限可达 $100 \mathrm{nmol} / \mathrm{L}$. 如 Eq. 19 所示，该纳米颗粒探针中的 $\mathrm{N}$ 原 子和两个羰基 $\mathrm{O}$ 原子与 $\mathrm{Fe}^{3+}$ 配位形成 $1: 1$ 型配合物, 而使探针 60 分子中的内酰胺开环出现苂光. 结合苂光 共聚焦显微成像技术，该纳米粒子探针可有效的检测活 细胞中 $\mathrm{Fe}^{3+}$ 浓度.

2012 年, 我们课题组 ${ }^{[48]}$ 也合成报道了系列罗丹明 噻唑基 $\mathrm{Fe}^{3+}$ 苂光探针 61 (Scheme 13), 并获得了探针与 $\mathrm{Fe}^{3+}$ 识别形成的配合物单晶. IR 光谱分析、 $\mathrm{NBO}$ 分析及 配合物单晶结构均表明 $\mathrm{Fe}^{3+}$ 与噻唑 $\mathrm{N}$ 原子的配位诱导

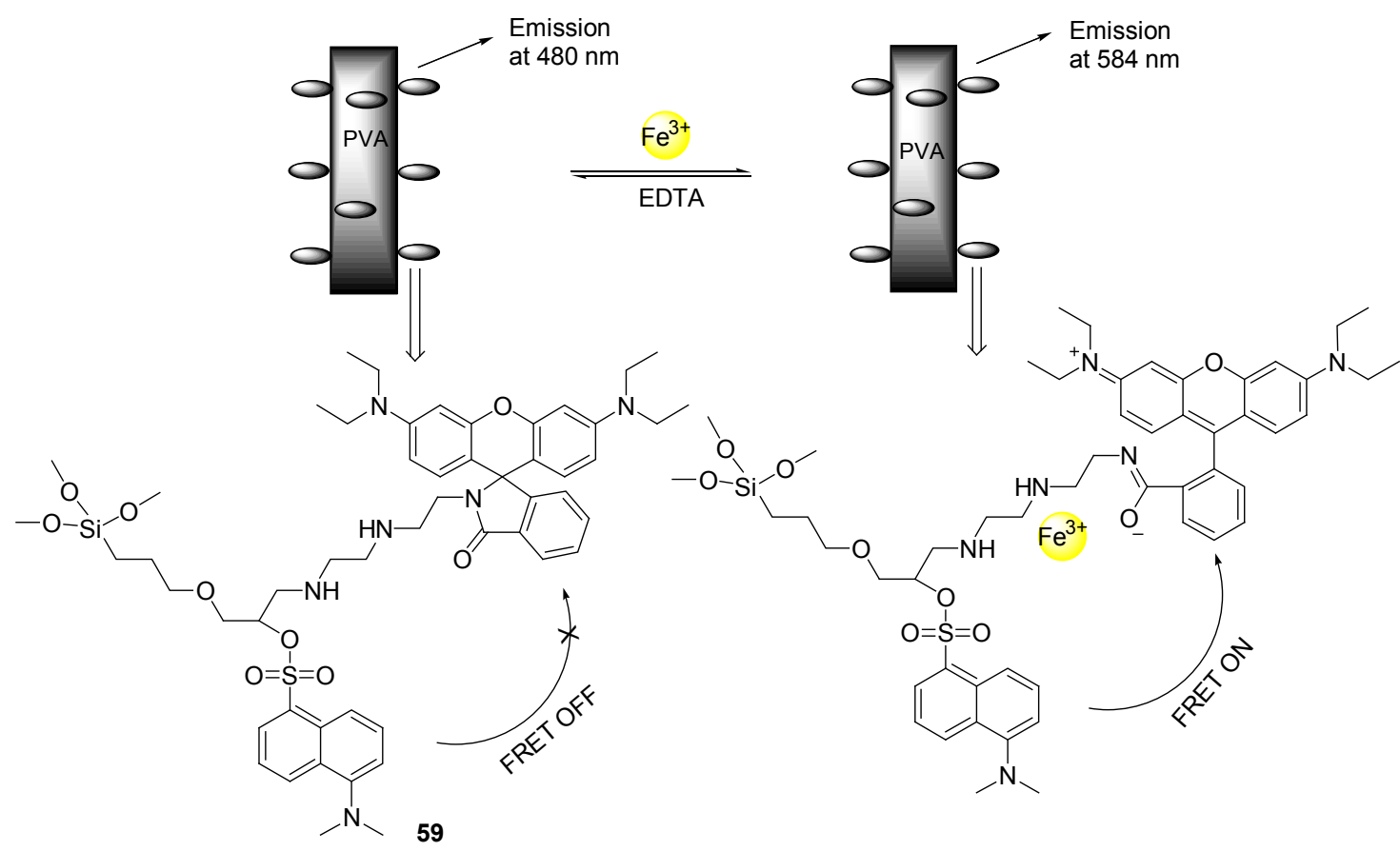




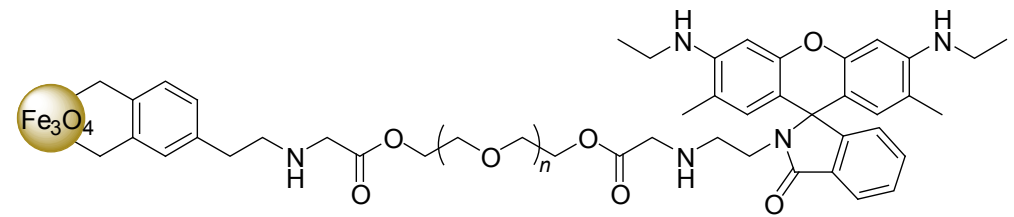

60

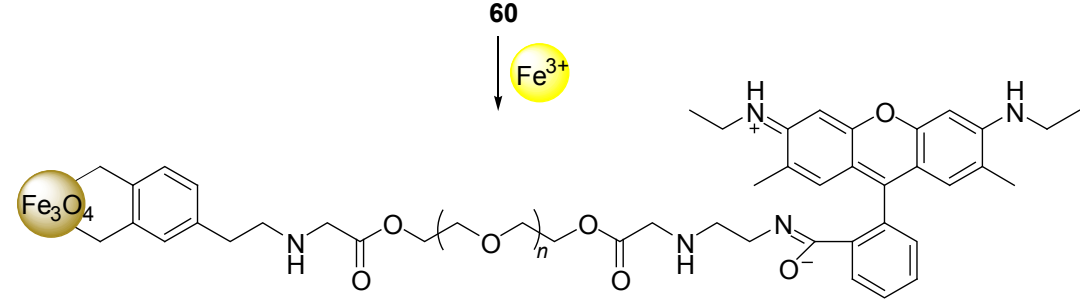
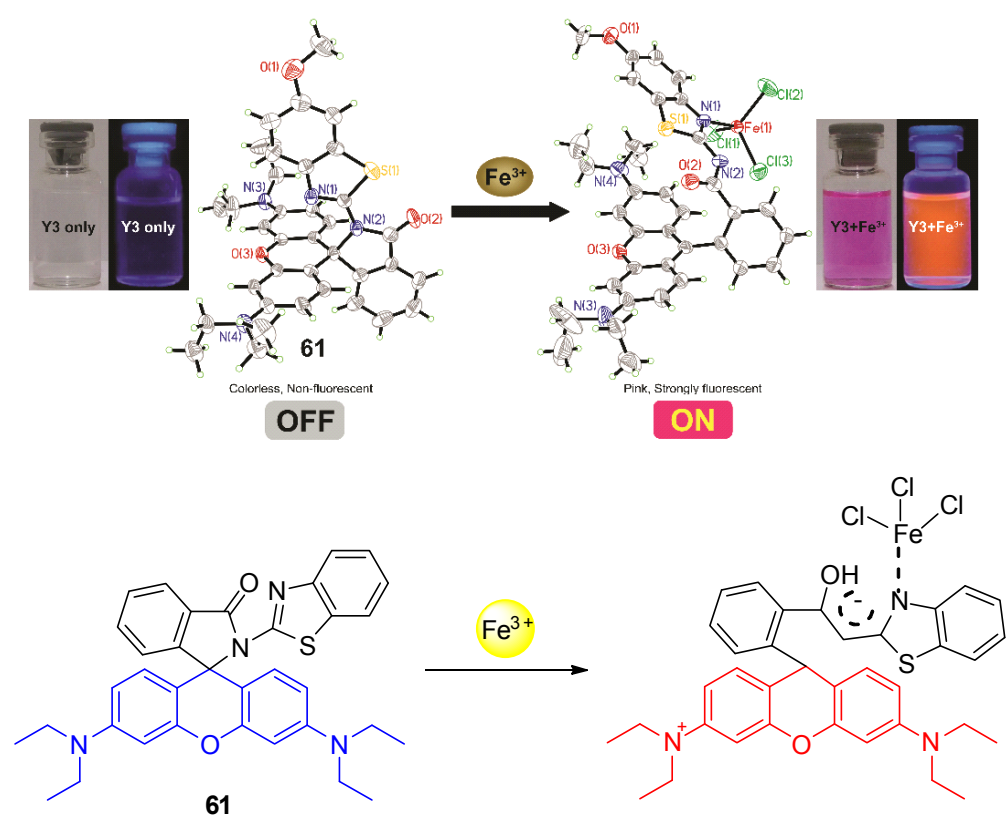

Scheme 13

罗丹明内酰胺开环进而产生苂光. 该系列探针合成过程 简便, 对 $\mathrm{Fe}^{3+}$ 表现出高灵敏度高选择性的检测, 且不受 其它共存离子的干扰，具有很好的应用前景.

2013 年, Thennarasu 等 ${ }^{[49]}$ 开发出一种单分子 FRET 基比率型 $\mathrm{Fe}^{3+}$ 探针 62 (Eq. 20), 其对 $\mathrm{Fe}^{3+}$ 显示出高选择 性响应, 检测线可达 $10 \mathrm{nmol} / \mathrm{L}$ 级别. 当向探针的水溶 液中加入 $\mathrm{Fe}^{3+}$ 时, 可看到明显的颜色和荧光变化, 可实 现比色法定性水溶液和生物样品中 $\mathrm{Fe}^{3+}$ 的检测. 该探针 在生理 $\mathrm{pH}$ 条件下性能稳定, 可实现活的 NIH3T3 细胞 中 $\mathrm{Fe}^{3+}$ 的检测. 这也是首例报道基于 FRET 机理的比率 型 $\mathrm{Fe}^{3+}$ 荧光探针, 具有较好的应用前景.

\section{$1.7 \mathrm{Cr}^{3+}$ 离子探针}

2011 年, Duan 等 ${ }^{[50]}$ 设计得到了一种功能化的硅纳 米材料, 并将其与罗丹明 $6 \mathrm{G}$ 衍生物结合得到另一种可 在水溶液中对 $\mathrm{Cr}^{3+}$ 进行检测的探针 63 (Eq. 21). 相比于 原有的罗丹明 $6 \mathrm{G}$ 衍生物, 63 不仅可在一定的 $\mathrm{pH}$ 条件下

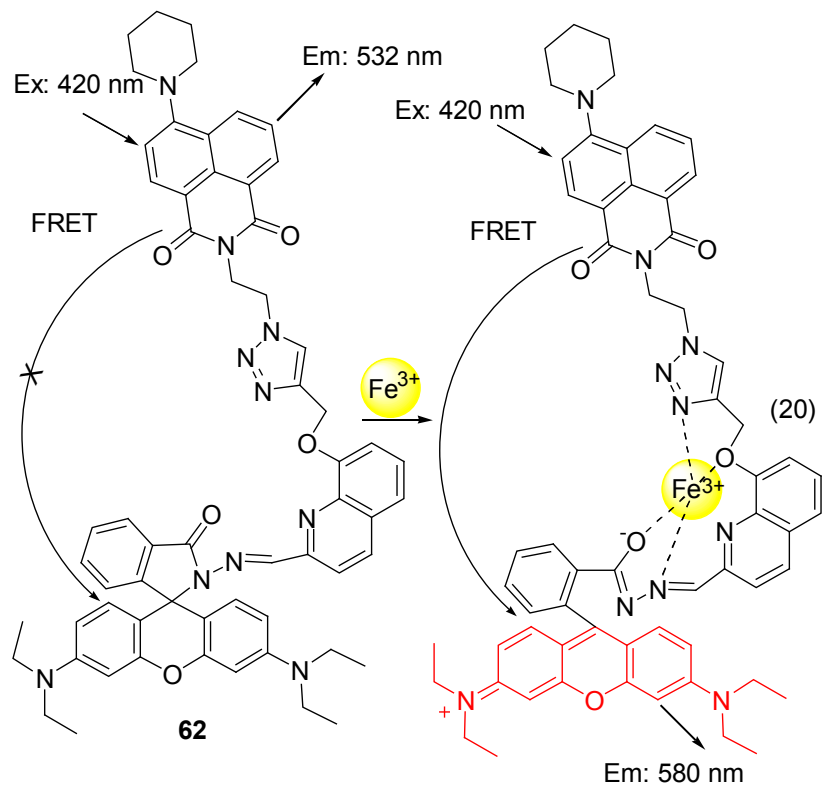

的水溶液中, 对 $\mathrm{Cr}^{3+}$ 高选择性和高灵敏度的可逆性检 
测, 还可通过硅纳米材料的空隙, 对水溶液中的 $\mathrm{Cr}^{3+}$ 进 行清除. 菼光成像实验表明该探针可进一步应用于活细 胞和组织中 $\mathrm{Cr}^{3+}$ 的检测.

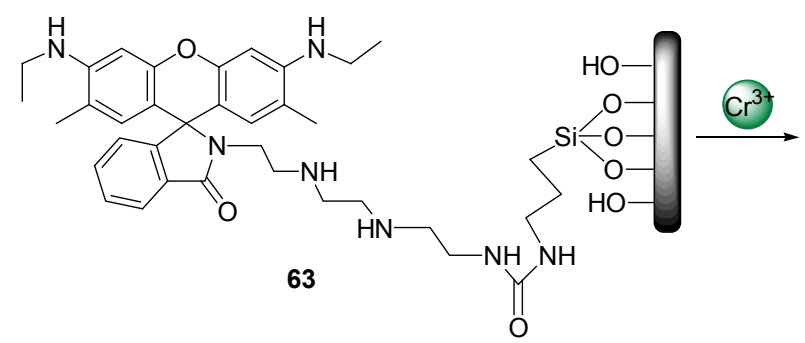

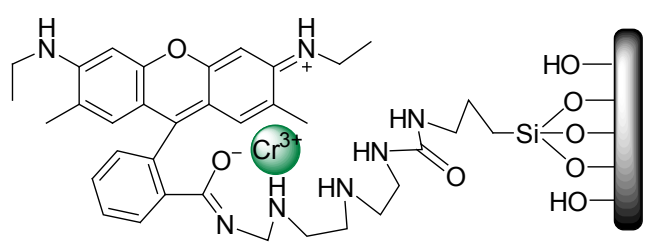

2012 年, $\mathrm{Pu}$ 等 $^{[51]}$ 报道了一种含有光敏变色的二芳 基乙烯和罗丹明基团的光致苂光信号转换探针 64 (Scheme 14), 该探针在光照下表现出明显的光致变色 和良好的抗衰减性能. 在 $\mathrm{EtOH}$ 溶液中, 当向探针中加 入 $\mathrm{Cr}^{3+}$ 时, 二芳基乙烯的颜色从无色变为紫色, 苂光发 射强度增强 300 倍. 当 $\mathrm{Cr}^{3+}$ 存在时, 在紫外光照射下, 可发生从能量供体罗丹明到能量受体二芳基乙烯基团 的 FRET 现象, 使探针回到闭环结构而荧光强度降低,
应用可见光照射则可使其荧光强度恢复. 这种具有多功 能响应的探针为今后研发新型多功能分子探针提供了 较好的参考模型.

2012 年, Das 等 ${ }^{[52]}$ 合成了两种可在干扰离子存在下 同时对 $\mathrm{Cr}^{3+}$ 和 $\mathrm{Hg}^{2+}$ 产生响应的探针 65 (Eq. 22), 该响应 在可见光范围内, 可通过肉眼进行观察. 探针 65 可作为 比率型探针通过 FRET 过程对 $\mathrm{Cr}^{3+}$ 和 $\mathrm{Hg}^{2+}$ 进行检测, 荧 光共聚焦成像实验表明该探针可用于 A431 细胞中金属 离子的检测.

\section{8 贵金属离子探针}

2009 年, Ahn 等 ${ }^{[53]}$ 报道了一种罗丹明 B 苂光比色探 针 66 (Eq. 23), 并首次应用该探针测定环境水体系中 $\mathrm{Ag}^{+}$和纳米银粒子含量. 在 $\mathrm{Ag}^{+}$存在的体系中, $\mathrm{Ag}^{+}$可与 66 中的碘结合形成噁唑啉环, 进而使内酰胺开环, 在氧 化条件下，纳米银粒子也可以发生这种开环过程. 该探 针不但对 $\mathrm{Ag}^{+}$表现出较高的选择性, 可用以测定最低 14 $\mu \mathrm{mol} / \mathrm{L}$ 浓度级别的含 $\mathrm{Ag}^{+}$溶液, 而且可对食品杀菌剂和 纤维软化剂中的纳米银粒子进行检测, 显示出较强的应 用前景.

2012 年, Peng 等 ${ }^{[54]}$ 合成了一种含有 [15] $\mathrm{aneNO}_{2} \mathrm{~S}_{2}$ 大 环配体的罗丹明衍生物 67 (Eq. 24). 该探针可以在纯水 溶剂中实现对 $\mathrm{Ag}^{+}$的快速苂光增强响应，检测线可达到 $100 \mathrm{nmol} / \mathrm{L}$. 同时，作者还通过理论计算首次证明了该 探针在乙腈和水溶剂中对 $\mathrm{Ag}^{+}$和 $\mathrm{Hg}^{2+}$ 的苂光响应过程
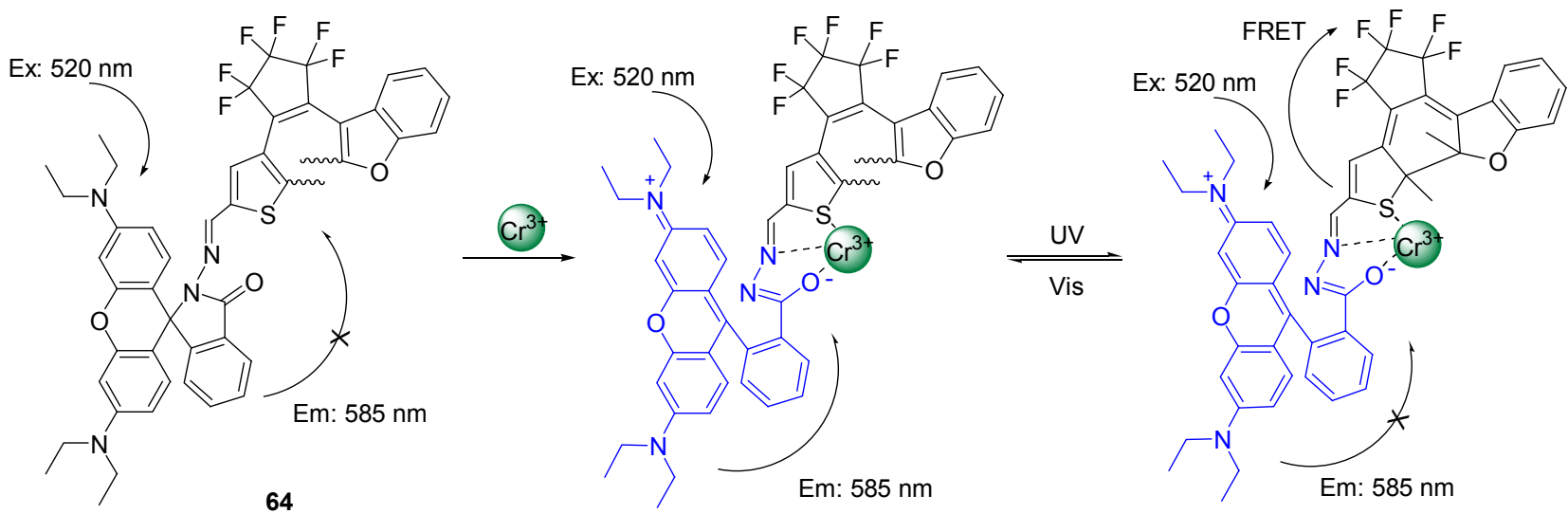

Scheme 14

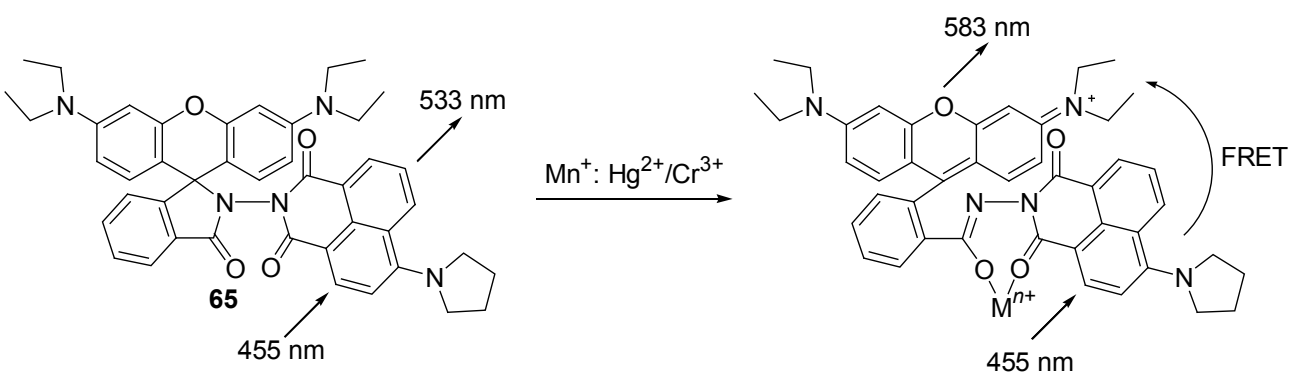




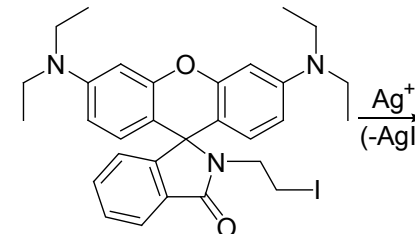

66<smiles></smiles>

(23)

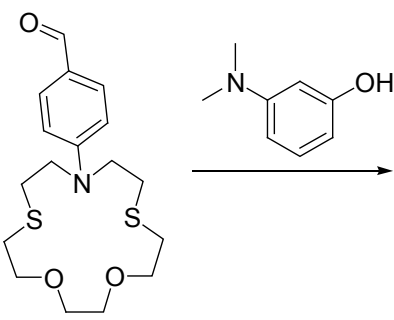

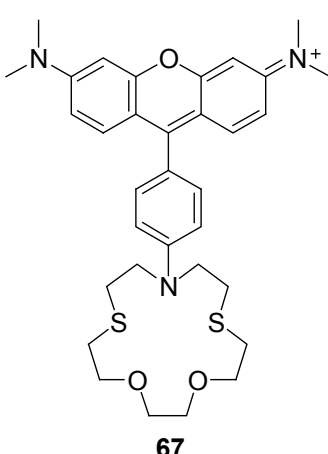

(24)
的区别, 研究表明该探针是通过 FRET 过程在水溶液中 对 $\mathrm{Ag}^{+}$进行识别. 细胞成像实验表明该探针具有较好的 细胞穿透性, 可用于活细胞中 $\mathrm{Ag}^{+}$的荧光成像.

Tae 等 ${ }^{[55]}$ 选择以罗丹明衍生物与含有炔基官能团的 物质作用, 其合成的衍生物作为检测探针可高效且快速 的识别 $\mathrm{Au}^{3+}$. 在水溶液中, 该探针对 $\mathrm{Au}^{3+}$ 表现高选择 性, 检测限可达 $50 \mathrm{nmol} / \mathrm{L}$. 该探针的识别机理是通过 与罗丹明相连的炔基, 在 $\mathrm{Au}^{3+}$ 诱导下与罗丹明内酰胺 发生不可逆的成环反应而使内酰胺开环产生荧光. 随 后, Yoon 和 Ahn 课题组 ${ }^{[56]}$ 又分别报道了类似的罗丹明 炔基化合物 68 (Scheme 15), 该探针也是利用催化活性 的 $\mathrm{Au}^{+} / \mathrm{Au}^{3+}$ 独特的 “亲炔性”, 使炔基进攻亲核的氧原 子, 并促使内酰胺开环及噁唑啉的形成. 这种基于催化 反应的探针对 $\mathrm{Au}^{+} / \mathrm{Au}^{3+}$ 具有很高的选择性和灵敏度. Yoon 等报道该探针可在 $\mathrm{pH}=7.4$ 条件通过肉眼对探针 的识别性能进行观察, 进而荧光共聚焦成像实验显示, 将 68 和 $\mathrm{HeCaT}$ 细胞共培养, 向其中加入 $\mathrm{Au}^{3+}$ 即可观察 到细胞内荧光增强, 表明该探针可用于活细胞中 $\mathrm{Au}^{3+}$ 的检测.

2012 年, Ahn 等 ${ }^{\left[{ }^{[7]}\right.}$ 设计报道了一类新型的反应型苂 光素基 $\mathrm{Au}^{3+}$ 探针 69 (Eq. 25). 该类探针分子中含有一个 炔基苯甲酸酯基团, 可以在 $\mathrm{Au}^{3+}$ 催化下发生酯水解反 应同时伴随苂光的产生而对 $\mathrm{Au}^{3+}$ 进行高灵敏度高选择 性识别.

2010 年, Tae 等 ${ }^{[58]}$ 报道了一种可在水溶剂体系下选 择性的检测 $\mathrm{Pt}^{2+}$ 的罗丹明三唑基荧光检测器 70 (Eq. 26). 作者通过 “点击反应” 将连有一个炔丙基的罗丹明 $6 \mathrm{G}$ Schiff 碱的炔基变为三唑基团. 在水溶液中, 这种具有 Schiff 碱和三唑两个官能团的探针对 $\mathrm{Pt}^{2+}$ 表现出较高的<smiles>C#CCN1C(=O)c2ccccc2C12c1ccccc1Oc1cc(N(CC)CC)cc(c1)C21C(=O)c2ccccc2Oc2cc(N(CC)CC)ccc21</smiles>

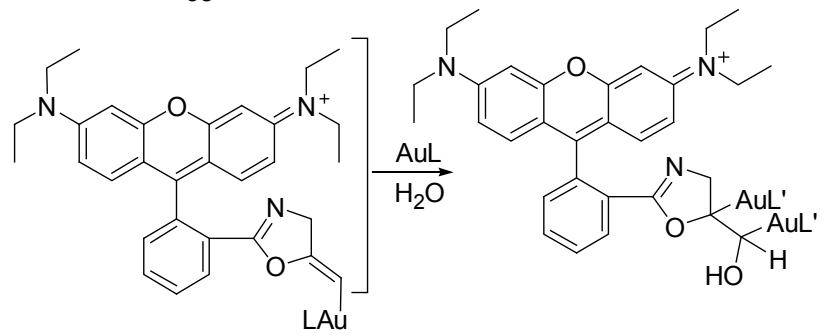<smiles>CCN(CC)c1ccc2c(c1)Oc1cc([N+](CC)(CC)CC)ccc1-c1ccccc1-2</smiles>

Scheme 15

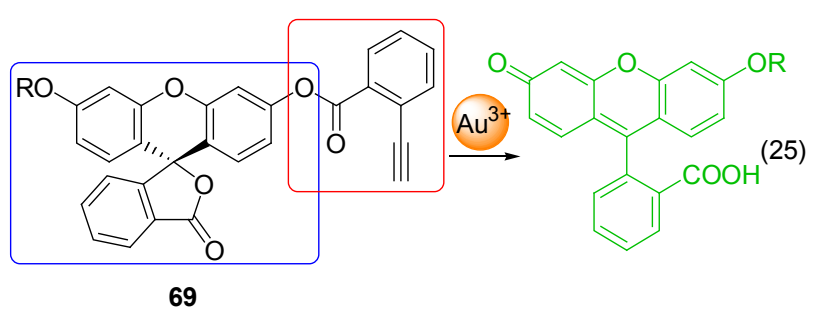

灵敏度和选择性, 探针可在较宽的 $\mathrm{pH}$ 范围内对 $\mathrm{Pt}^{2+}$ 表 现出可逆性检测，该荧光探针已被应用于水溶液中顺铂 的检测.
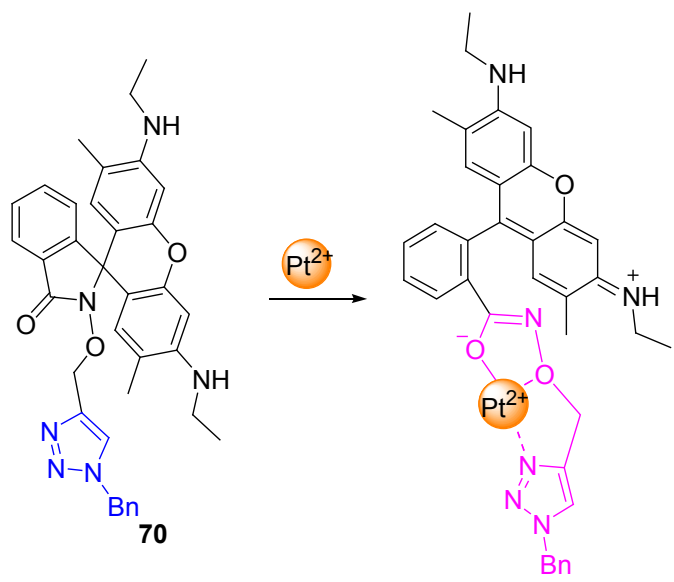

同年, $\mathrm{Ahn}$ 等 ${ }^{[59]}$ 报道了一种可有效的对 $\mathrm{Pd}^{2+}$ 进行光 学识别的罗丹明衍生物 71 (Scheme 16). 该探针的识别 机理为: 在探针 71 中氧化性的插入 $\mathrm{Pd}^{0}$ 形成中间体 71-I, 进而酰胺中 $\mathrm{O}$ 和 $\mathrm{Pd}$ 之间的络合作用使罗丹明内酰胺开 
环而使该中间体发生转化形成 71-II, 最后发生还原消 除形成苯并噁唑，同时罗丹明基团产生荧光. 该探针对 $\mathrm{Pd}^{2+}$ 具有极高的选择性, 可用于化学品中剩余 $\mathrm{Pd}$ 含量 的检测, 检测限可达 $30 \mu \mathrm{mol} / \mathrm{L}$. 随后, Peng 等 ${ }^{\left[{ }^{[0]}\right.}$ 相继报 道了多种高选择性的 $\mathrm{Pd}^{2+}$ 荧光探针. 2012 年, 该研究 组 ${ }^{[61]}$ 开创性的设计合成了一种含有三齿 PNO 结合集团 的新型 $\mathrm{Pd}^{2+}$ 探针. 由于分子中的三齿 PNO 结合基团具 有很强的配位能力, 因此该探针具有较快的反应时间和 较高的灵敏度和选择性, 性能较之前报道的多种探针提 升很多. 该探针对 $\mathrm{Pd}^{2+}$ 和 $\mathrm{Pd}^{0}$ 不同的荧光响应情况将有 助于研究不同价态 Pd 物质的选择性状况. 目前该探针 成功应用于药物、水、土壤和植物叶子中 Pd 的转移分 析, 这将有助于研究 Pd 的环境行为以避免该贵金属和 有害金属对环境的损害.

2013 年, Liu 等 ${ }^{[62]}$ 合成报道了双炔丙基相连的罗丹 明基 $\mathrm{Pd}^{2+}$ 苂光探针 72 (Scheme 17). 在 $\mathrm{Pd}^{2+}$ 存在下, 72 的荧光强度增强超过 113 倍, 相比于其它金属离子, 72 对 $\mathrm{Pd}^{2+}$ 表现出较高的特异选择性. 在 $\mathrm{Pd}^{2+}$ 氛围中, 探针
的炔丙基离去使内酰胺开环产生荧光，该过程已经通过 红外光谱和合成共振光谱证实. 同时, 72 还可在没有还 原剂存在下检测 $\mathrm{Pd}^{0}, 72-\mathrm{Pd}^{0}$ 的苂光发射强度和 $72-\mathrm{Pd}^{2+}$ 基本相同，其应用前景广阔.

\section{9 其它金属离子探针}

2009 年, Duan 等 ${ }^{[63]}$ 设计合成了一种含有罗丹明 $6 \mathrm{G}$ 和 8-羟基喹啉基团的发光探针 73 (Eq. 27), 在可见光区, 该探针可将能量传递给 $\mathrm{Yb}^{3+}$, 进而在 $1000 \mathrm{~nm}$ 附近产生 近红外发射. $\mathrm{Yb}^{3+}$ 诱导罗丹明 $6 \mathrm{G}$ 内酰胺开环使其在 525 $\mathrm{nm}$ 处出现紫外吸收并在 $560 \mathrm{~nm}$ 产生荧光发射. 在此过 程中，8-差基喹啉基团失去一个质子，使探针与 $\mathrm{Yb}^{3+}$ 形 成四配位配合物.

2012 年, $\mathrm{Yi}$ 等 ${ }^{[64]}$ 设计合成了一种可对 $\mathrm{Sn}^{4+}$ 进行可 逆性检测的罗丹明-菜二甲酰亚胺基探针 74 , 这也是首 次报道的可用于 $\mathrm{Sn}^{4+}$ 检测的荧光探针(Eq. 28). 利用该 探针可实现对生物体内的可用于 $\mathrm{Sn}^{4+}$ 检测的荧光探针 (Eq. 28). 利用该探针可实现对生物体内 $\mathrm{Sn}^{4+}$ 的检测, 很

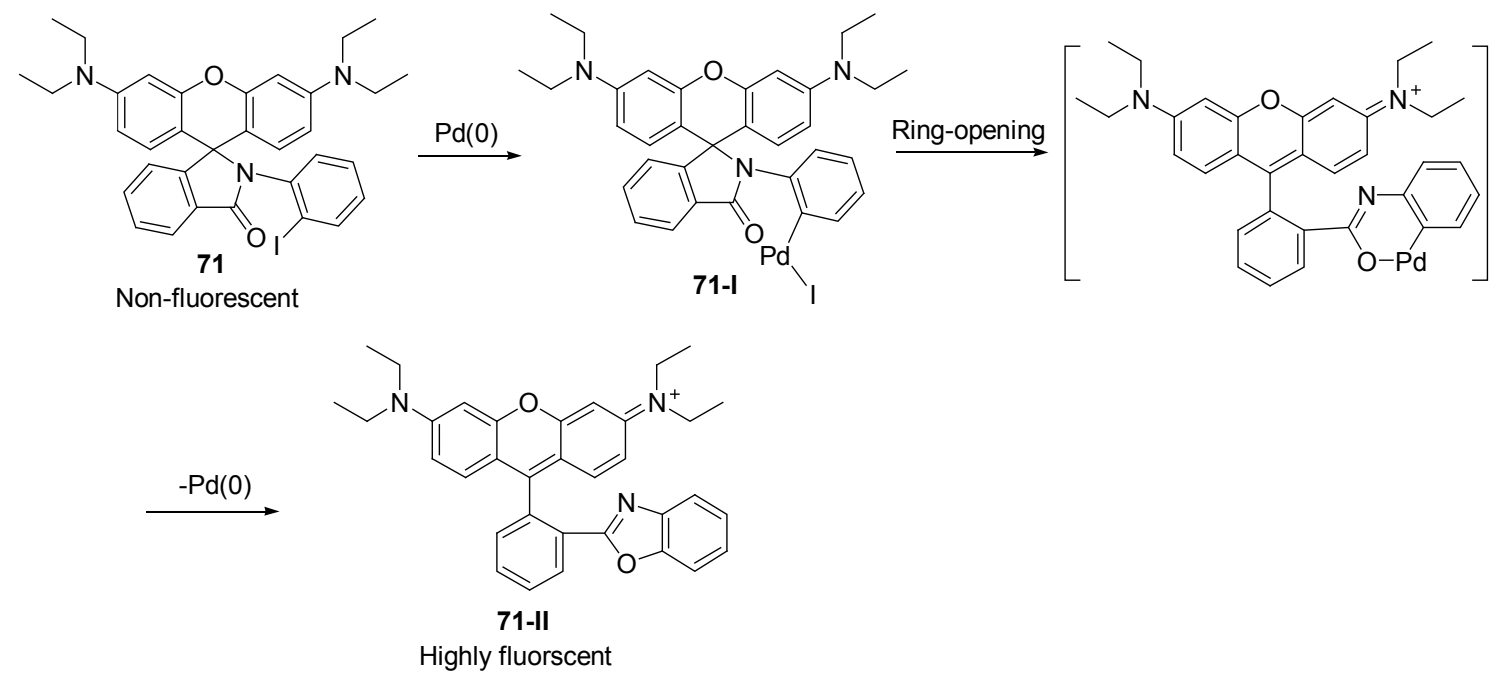

Scheme 16<smiles>C#CCN(CC#C)CCN1C(=O)c2ccccc2C12c1ccc(N(CC)CC)cc1Oc1cc(N(CC)CC)ccc12</smiles>

72

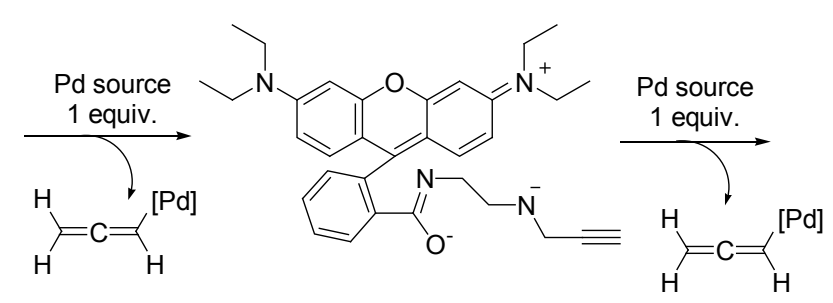<smiles>CCN(CC)c1ccc2c(-c3ccccc3C(O)=NCC[NH-])c3ccc(=[N+](CC)CC)cc-3oc2c1</smiles>

Scheme 17 
<smiles>CCNc1cc2c(cc1C)C1(c3cc(C)c(NCC)cc3O2)c2ccccc2C(=O)N1/N=C/c1ccc2cccc(O)c2n1</smiles>

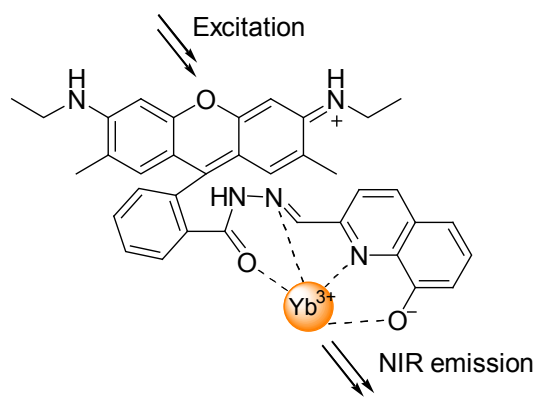

大地促进了对 $\mathrm{Sn}^{4+}$ 在生物系统中作用的研究.
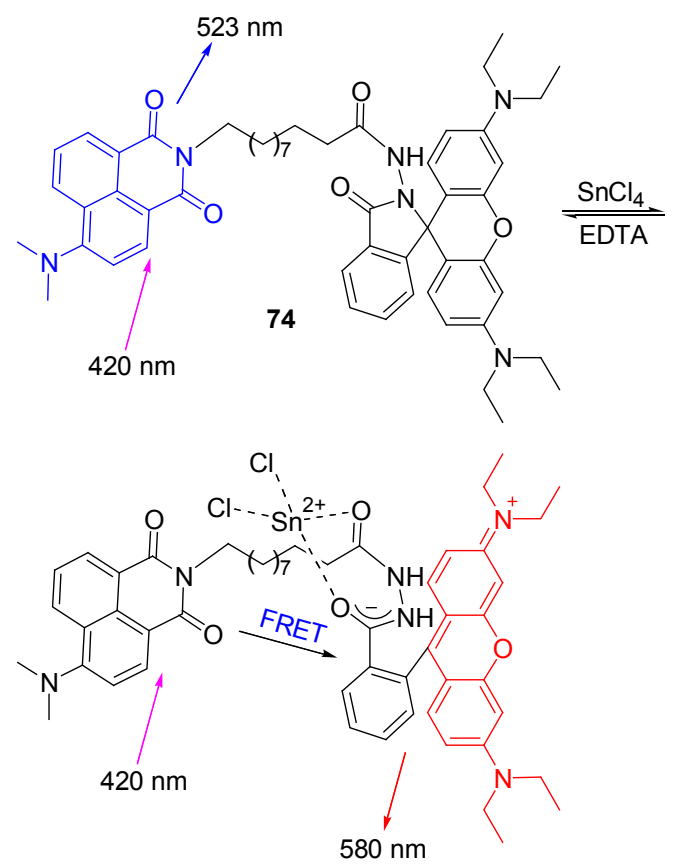

(28)

\section{2 基于氧杂葱环的 $\mathrm{pH}$ 探针}

2011 年, Han 等 ${ }^{[65]}$ 首次报道介孔氧化硅溶酶体 $\mathrm{pH}$ 比色探针 75 (Eq. 29), 该探针分子中包含能被细胞溶酶 体活化的罗丹明内酰胺基团, 可以在单波长激发下通过 荧光共聚焦成像和流式细胞术对活细胞中的 $\mathrm{pH}$ 进行检 测. 溶酶体 $\mathrm{pH}$ 的改变与多种细胞响应方式有关, 可通 过检测溶酶体内在的酸度改变产生的荧光信号对小鼠 癌细胞进行荧光成像分析. 该纳米探针的合成报道，对 溶酶体相关的细胞生物学领域的探索以及溶酶体靶向 癌症治疗研究的推进提供了一种新的技术方法.

2012 年, Ma 等 ${ }^{[66]}$ 通过将 FITC(荧光素)和 RBITC(罗

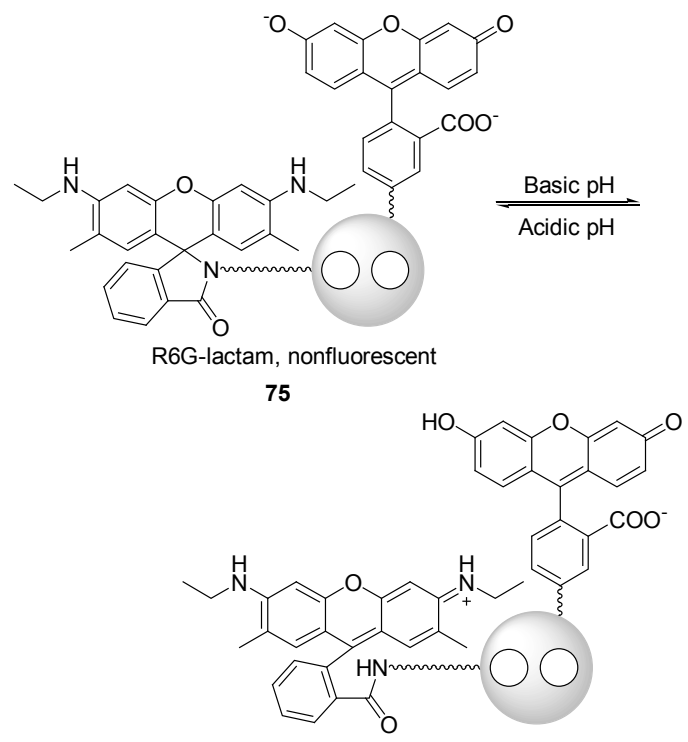

R6G-amide, fluorescent

丹明)合并到碳纳米点上，得到了一种可调控的纳米 $\mathrm{pH}$ 荧光探针 76 (Scheme 18), 其显著的特点是可以很容易 的实现对 $\mathrm{pH}$ 响应的调控. 细胞成像实验表明该探针具 有较好的生物相容性和细胞内分散性，可定量检测完整 的 HeLa 细胞中 $\mathrm{pH}$ 和由氧化刺激所造成的 $\mathrm{pH}$ 波动. 这 项研究工作的深入推进，证实了 $\operatorname{CDs}$ (碳纳米点)有希望 成为实用苂光纳米探针良好的设计平台，也有希望实现 不同刺激下分子内 $\mathrm{pH}$ 的定量检测.

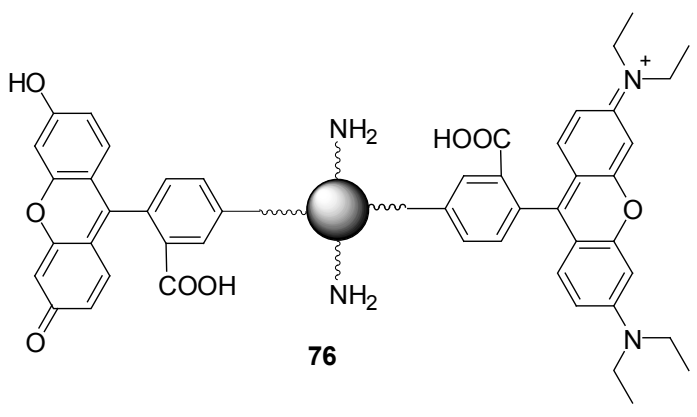

Scheme 18

Peng 等 ${ }^{[67]}$ 在随后的 2012 年, 设计了一种高灵敏度 高选择性 $\mathrm{pH}$ 探针 77 (Eq. 30), 其分子中含有细胞溶酶 体定位基团二甘醇一甲醚, 可有效的对细胞溶酶体进行 染色. 该探针的染色性能是因为二甘醇一甲醚基团的存 在，以此可有效的避免目前细胞溶酶体探针中含氮侧链 对细胞溶酶体的碱性化效应. 探针 77 具有较低的细胞 毒性和较好的生理适应性，可用于检测活细胞中的细胞 溶酶体 $\mathrm{pH}$, 亦可检测由氯喹引起的细胞溶酶体 $\mathrm{pH}$ 升高 和细胞死亡周期内溶酶体的酸性变化，显示出极强的生 物应用前景 
<smiles>[Z7]C[C@H](O)[C@H](C)O</smiles><smiles>CCN(CC)c1ccc2c(-c3ccccc3C(=O)NC(=O)COCCOCCOC)c3ccc(=[N+](CC)CC)cc-3oc2c1</smiles>

\section{3 基于氧杂葱环的阴离子探针}

\section{1 次氯酸/次氯酸盐探针}

2011 年, Guo 等 ${ }^{[68]}$ 报道了一种可在不同溶剂中分别 对 $\mathrm{Cu}^{2+}$ 和 ${ }^{-} \mathrm{OCl}$ 产生高选择性和高灵敏度识别的苂光探 针 78 (Scheme 19), 其分子包含一个可以和 $\mathrm{Cu}^{2+}$ 配位的 吡啶甲酰胺基团和一个可以和 ${ }^{-} \mathrm{OCl}$ 发生反应的二酰基 肼基团. 在 $\mathrm{CH}_{3} \mathrm{CN} / \mathrm{H}_{2} \mathrm{O}(V / V=9 / 1,10 \mathrm{mmol} / \mathrm{L}$, Tris- $\mathrm{HCl}$, $\mathrm{pH} 7.0$ ) 溶液中, 78 可对 $\mathrm{Cu}^{2+}$ 表现出可逆性的检测并且产 生荧光和颜色变化, 而在 $\mathrm{CH}_{3} \mathrm{CN} / \mathrm{H}_{2} \mathrm{O}(V / V=7 / 3,30$ $\mathrm{mmol} / \mathrm{L}, \mathrm{Na}_{2} \mathrm{~B}_{4} \mathrm{O}_{7} / \mathrm{NaOH}, \mathrm{pH}$ 12)溶液中, 78 则对 ${ }^{-} \mathrm{OCl}$ 显 示出不可逆性的识别作用生成罗丹明 $\mathrm{B}$ 而使探针分子 产生荧光. 该探针是目前首例报道的可用于 $\mathrm{Cu}^{2+}$ 和 $\mathrm{OCl}$ 检测的小分子双功能探针, 检测限可达 $1 \mathrm{nmol} / \mathrm{L}$ 级别.

2012 年, $\operatorname{Lin}$ 等 $^{[69}$ 基于罗丹明-氨基硫脲可以在 $\mathrm{HClO}$ 催化下生成罗丹明-噁二唑的反应, 设计合成了两 种 FRET 型的 $\mathrm{HClO}$ 探针 79 (Eq. 31), 这也是首次报道 的 FRET 型的 $\mathrm{HClO}$ 探针. 这两种探针灵敏度高、选择 性好、反应快速, 在生物 $\mathrm{pH}$ 下具有良好的性能, 毒性较 低且极易透过细胞膜. 此外, 其良好的比率探针性能也 使其有望成为研究 $\mathrm{HClO}$ 生物行为的有力工具, 同时使 用 $\mathrm{HClO}$ 催化罗丹明-氨基硫艮环化生成罗丹明-噁二唑 的设计思路也将为今后设计更多性能优良的反应型 $\mathrm{HClO}$ 探针提供新的构想.

2012 年, Peng 等 ${ }^{[70]}$ 利用氨基青蓝染料对 ROS 的响 应设计得到了一种可用于识别 ${ }^{-} \mathrm{OCl}$ 的比率型纳米苂光 探针 $\mathbf{8 0}$ (Scheme 20). 探针 $\mathbf{8 0}$ 对 ${ }^{-} \mathrm{OCl}$ 表现出极好的比率 型苂光响应, 成功应用于活细胞中 ${ }^{-} \mathrm{OCl}$ 的检测, 这也是 首次报道的可用于检测 ${ }^{-} \mathrm{OCl}$ 的比率型 FRET 荧光探针. 该探针是通过将两种有机染料简单的结合在硅纳米材 料上获得的, 而纳米材料本身优良的性能, 如较长的发 光时间和较好的生物适应性, 也预示着该探针良好的应 用前景. 该设计思路为进一步研发应用于生物系统的 FRET 比率型探针和多通道探针提供了绝佳的借鉴参

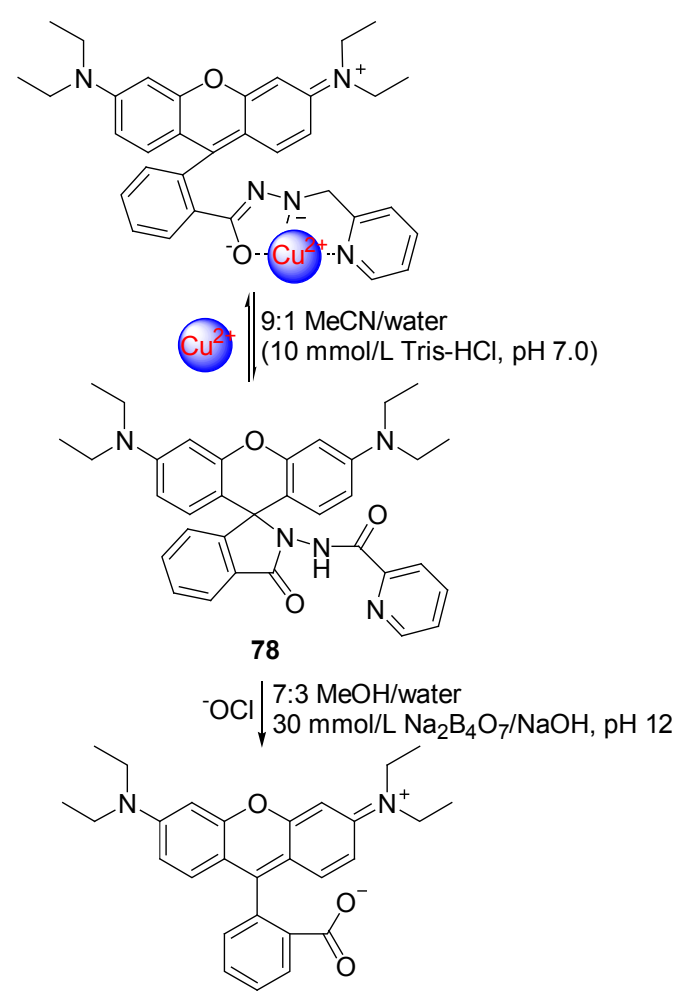

Scheme 19<smiles>CCN(CC)c1ccc2c(c1)Oc1cc(N(CC)CC)ccc1C21c2ccc(N3CCN(C(=O)c4cc5ccc(N(CC)CC)cc5oc4=O)CC3)cc2C(=O)N1NC(=S)Nc1ccccc1</smiles><smiles></smiles>

考.

2013 年, 我们课题组 ${ }^{[71]}$ 设计报道了可用于检测 ${ }^{-}$ $\mathrm{OCl}$ 的荧光素类 Schiff碱荧光探针 $\mathbf{8 1}$ (Scheme 21). 研究 发现，该系列探针是利用不可逆的 ${ }^{-} \mathrm{OCl}$ 的氧化作用来 实现苂光的 “off-on” 转变, 具有高选择性和高灵敏性等 优点，目前已成功应用于环境水质中 ${ }^{-} \mathrm{OCl}$ 的检测.

\section{$3.2 \mathrm{CN}^{-}$离子探针}

2011 年, Guo 等 ${ }^{[72]}$ 报道了一种可在水溶液中对 $\mathrm{CN}^{-}$ 表现出高选择性高灵敏度识别的 FRET 探针 82 (Scheme 22). 该探针分子中含有一个 4-(N,N-二甲基)苯甲酰胺基 才作为能量供体，而荧光素基团作为能量受体. $\mathrm{CN}^{-}$的 


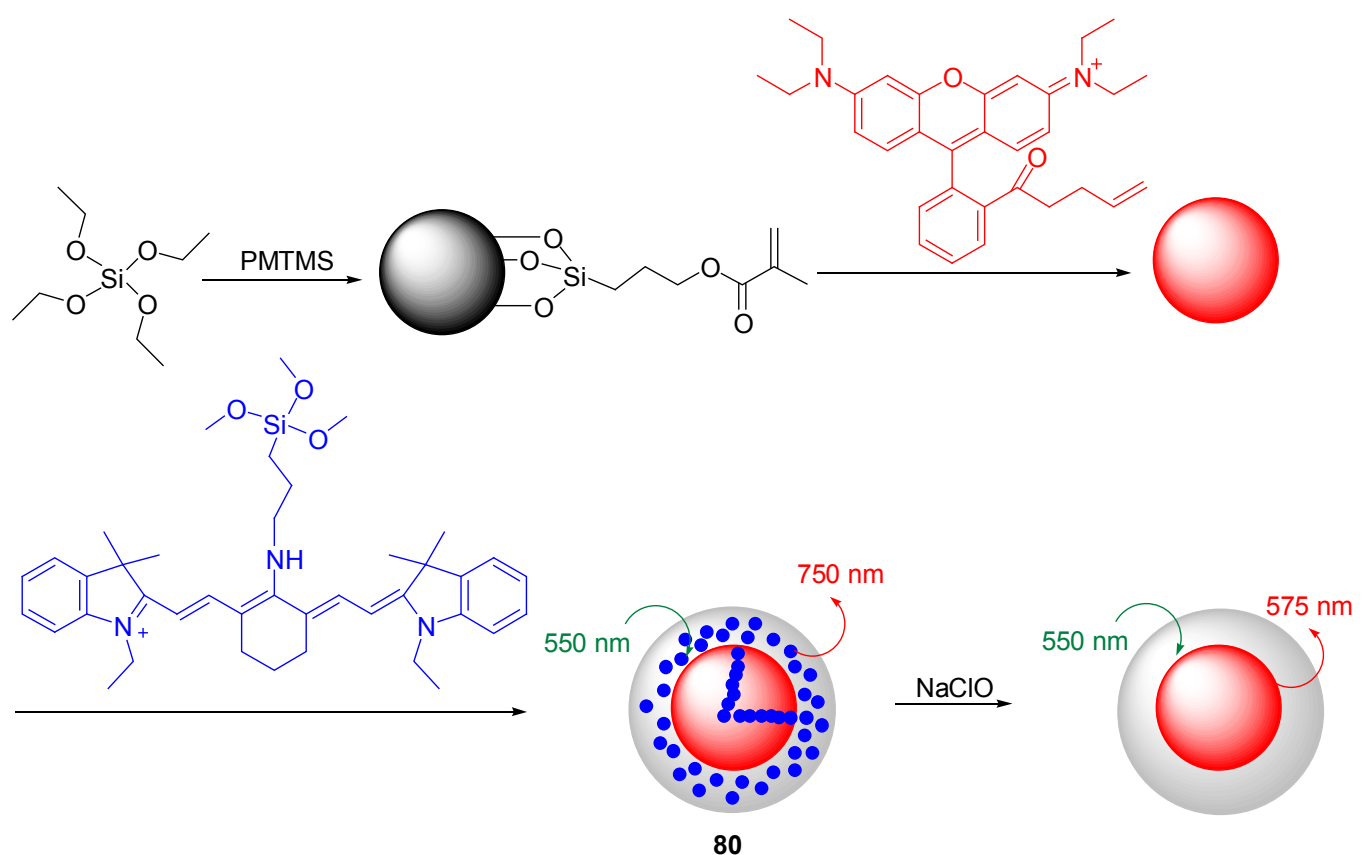

Scheme 20
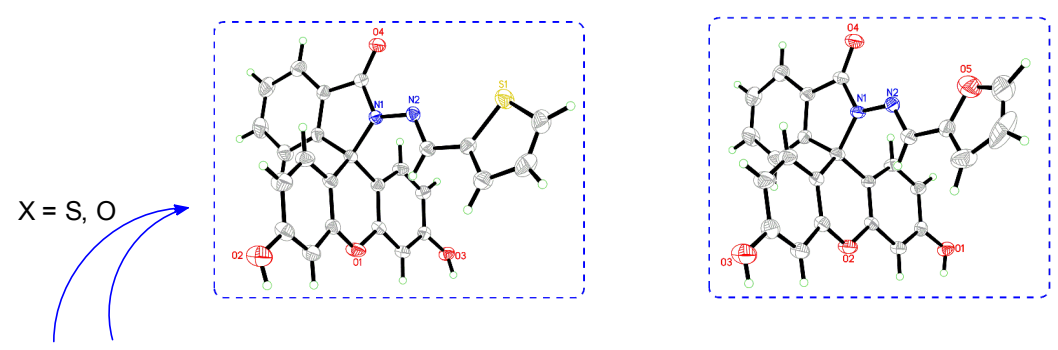

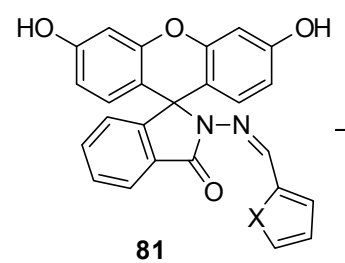

Colorless, non-fluorescent
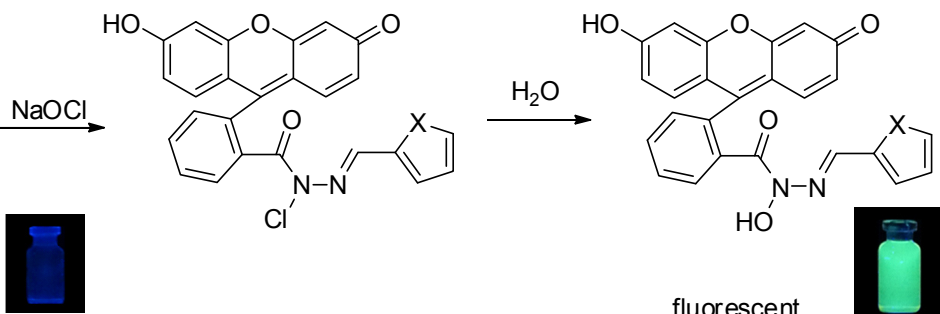

Scheme 21

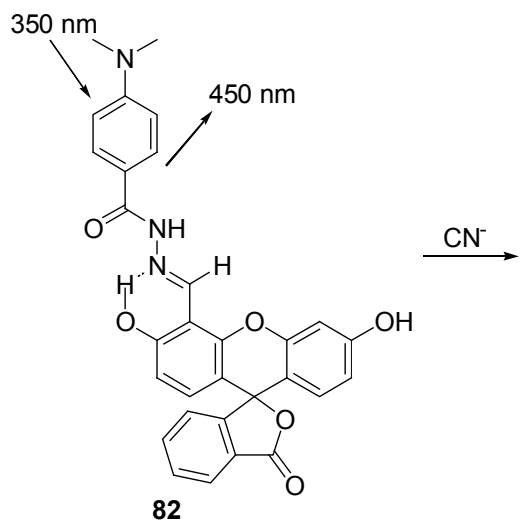

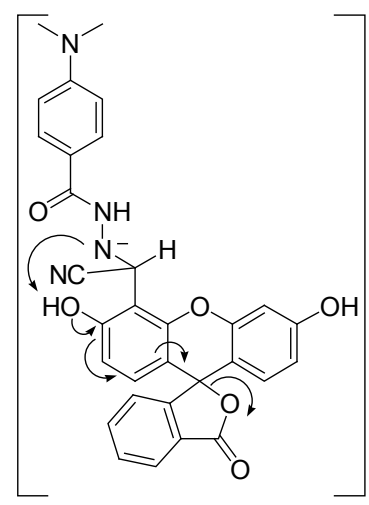

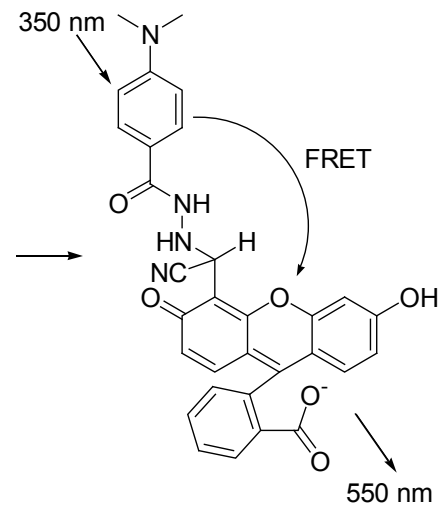

Scheme 22 
加入可使分子中 4-( N,N-二甲基)苯甲酰胺和苂光素两个 基团荧光强度增强, 并伴随溶液颜色的改变, 其它离子 对检测的干扰较小. 工作曲线显示探针和 $\mathrm{CN}^{-}$可形成 $1: 1$ 型加合物, 检测限可达 $44 \mu \mathrm{mol} / \mathrm{L}$, 其灵敏度足以 检测国际卫生组织所设定的饮用水中 $\mathrm{CN}^{-}$的浓度.

2012 年, Shiraishi 等 ${ }^{[73]}$ 报道了一种可在光照条件下 定量检测水溶液中 $\mathrm{CN}^{-}$的比色探针 83 (Scheme 23). 在 黑暗条件下, 探针 83 处于闭环状态而无苂光, 当用紫外 光照射时, 83 将变为开环异构体并在 467 和 $568 \mathrm{~nm}$ 处出 现荧光发射. 而向此溶液中加入 $\mathrm{CN}^{-}$后, $\mathrm{CN}^{-}$将会和开 环探针发生亲核反应, 上述两个荧光发射强度将会降低 而在 $512 \mathrm{~nm}$ 处出现新的荧光发射. 探针和 $\mathrm{CN}^{-}$可形成 $1: 1$ 型加合物, 在 $5 \sim 150 \mu \mathrm{mol} / \mathrm{L}$ 浓度范围内, 探针的 荧光强度和 $\mathrm{CN}^{-}$的浓度成正比, 可用于水溶液中 $\mathrm{CN}^{-}$的 定量检测，检测限为 $5.0 \mu \mathrm{mol} / \mathrm{L}$.

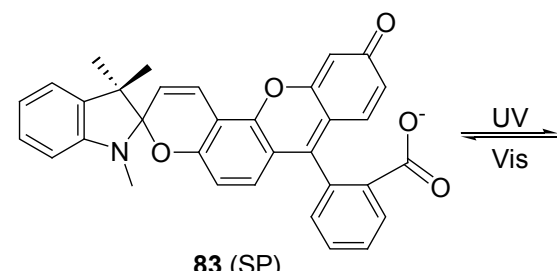

$83(\mathrm{SP})$

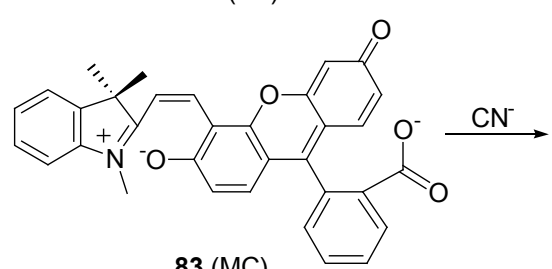

$83(\mathrm{MC})$

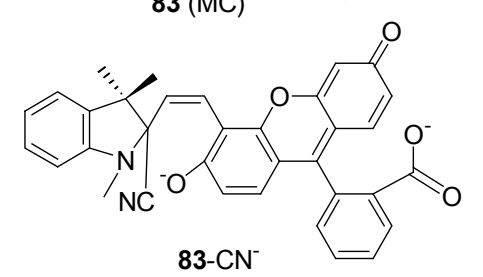

Scheme 23

同年, Guo 等 ${ }^{[74]}$ 设计了一种 $2: 2$ 型罗丹明 $-\mathrm{Cu}^{2+}$ 配 合物探针 84 (Eq. 32), 在该探针分子中, 罗丹明分子和 $\mathrm{Cu}^{2+}$ 形成接近平面的结构. 当加入 $\mathrm{CN}^{-}$时, $\mathrm{CN}^{-}$占据两 个 $\mathrm{Cu}^{2+}$ 的轴向配位位置而使分子产生较强的苂光发射 以实现对 $\mathrm{CN}^{-}$的高选择性和高灵敏度识别检测, 这些已 经通过 $X$ 射线单晶衍射得到证明. 虽然进一步的检测机 理仍有待研究, 但该研究为设计配合物探针以实现 $\mathrm{CN}^{-}$ 的检测提供了良好的模板.

2013 年, Lee 等 ${ }^{[75]}$ 设计了一种新型氧杂葱基荧光探 针 85 (Eq. 33), 其分子中甲酰基胍基团和醛基基团通过 分子结构凹面所产生的分子内氢键而连接到氧杂葱发 色团上. 探针分子中这种相互交错的氢键为分子内键的 旋转增加了显著的能量, 促进了探针分子结构的预组合

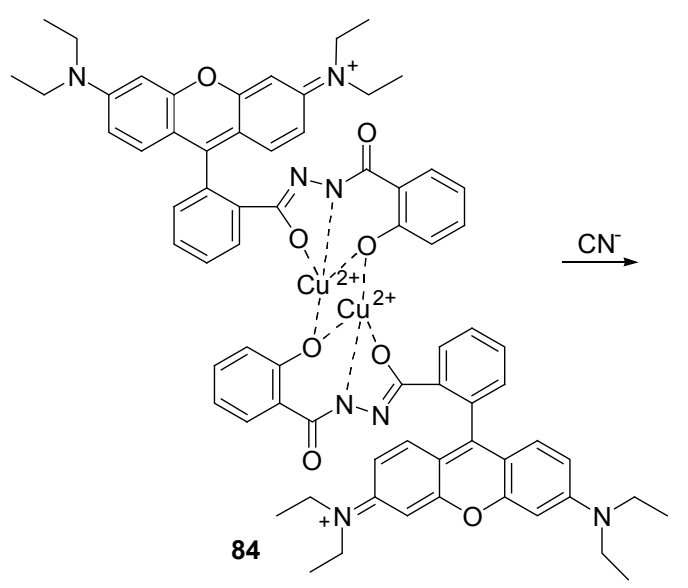

Weak fluorescence

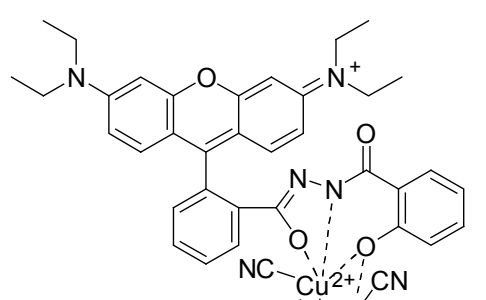

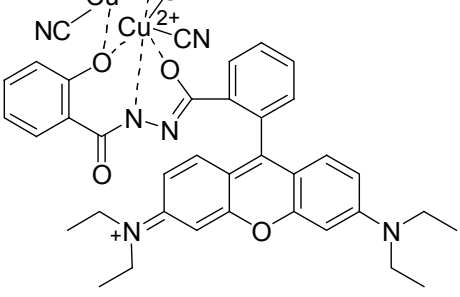

Strong fluorescence

并有效的极化羰基基团使其更容易受到进攻而进行识 别. 这种新颖的设计构思为合成性能更优的探针提供了 新的设计思路，具有很好的创新性.
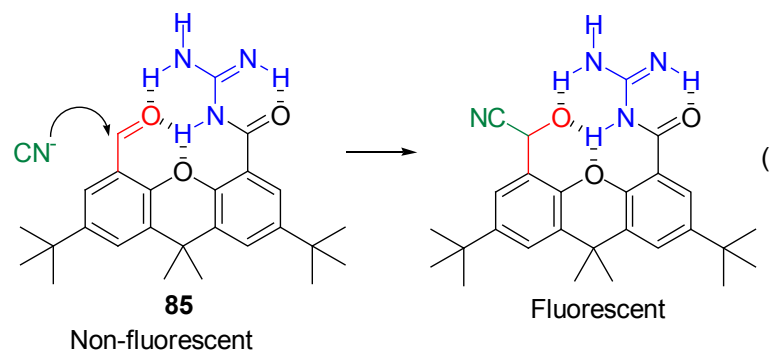

\section{3 $\mathrm{S}^{2-}$ 和 $\mathrm{H}_{2} \mathrm{~S}$ 探针}

应用荧光素酯在 $S^{2-}$ 或 $\mathrm{H}_{2} \mathrm{~S}$ 作用下反应生成苂光素, 是 $\mathrm{S}^{2-}$ 和 $\mathrm{H}_{2} \mathrm{~S}$ 探针普遍的设计思路(Scheme 24). 2009 年, Yang 等 ${ }^{[76]}$ 报道在含水的丙酮溶液中将探针 86 和 $\mathrm{S}^{2-}$ 混 合，会使 2,4-二硝基苯磺酰基团离去而使荧光素基团产 生明显的荧光发射，该探针已成功应用于工业废水中 $\mathrm{S}^{2-}$ 的检测. 2011 年, Xian 等 ${ }^{[77]}$ 报道的荧光素衍生物 87 可以在中性条件下受 $\mathrm{H}_{2} \mathrm{~S}$ 诱导生成苯并二硫酮, 应用此 可实现在水溶液、血液和细胞中有效的选择性检测 $\mathrm{H}_{2} \mathrm{~S}$, 


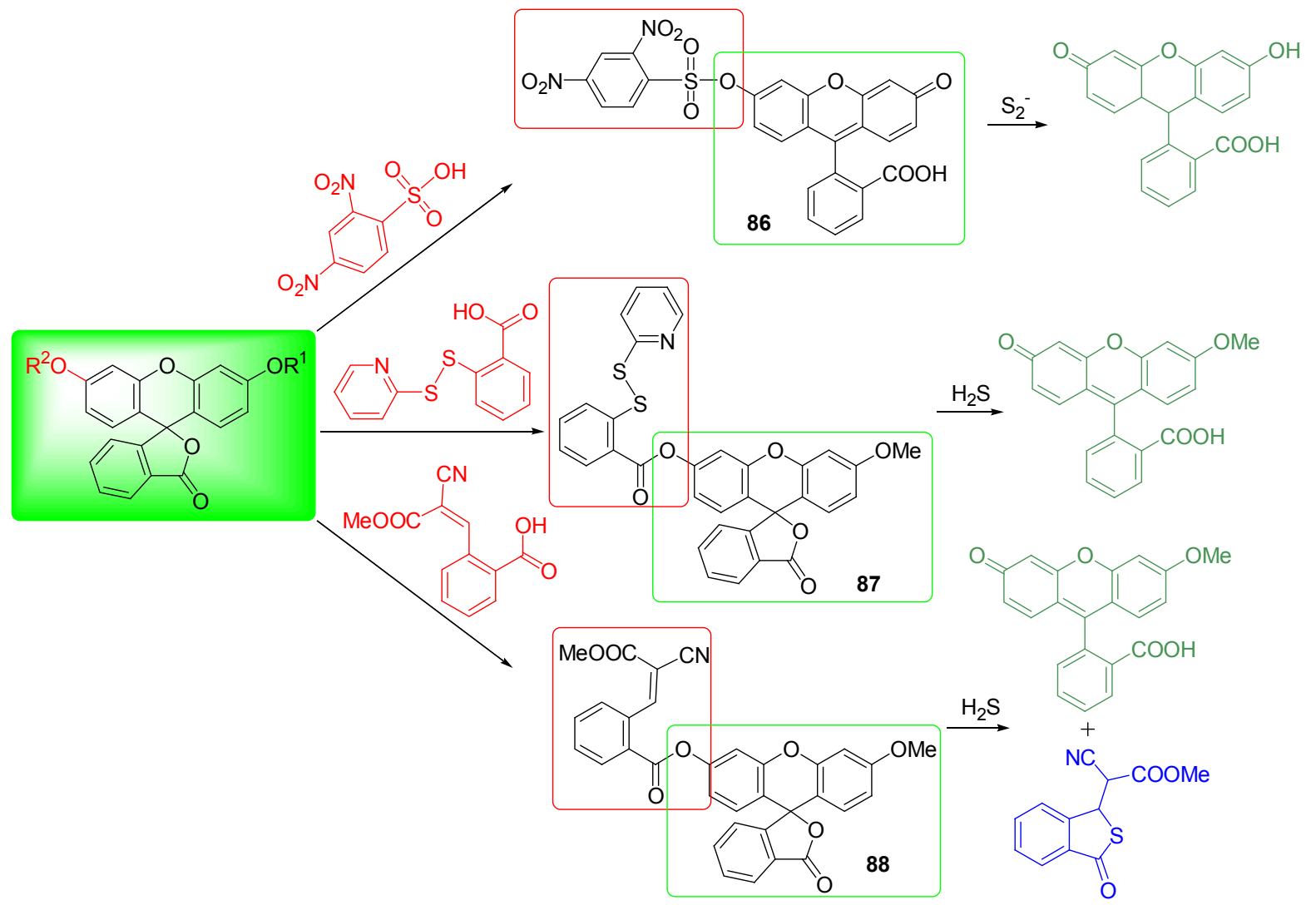

Scheme 24

其它生物硫醇物质如 Cys 和 Glu 等均不会产生干扰响应. 在此检测体系中, 不仅可以应用探针的荧光强度变化来 检测 $\mathrm{H}_{2} \mathrm{~S}$ 的浓度, 也可通过产物苯并二硫酮的产量来进 行分析检测. 2012 年, Xian 等 ${ }^{[78]}$ 又在此基础上应用 $\mathrm{H}_{2} \mathrm{~S}$ 参与迈克尔加成环化反应原理合成了另一种荧光素基 “off-on” 型 $\mathrm{H}_{2} \mathrm{~S}$ 探针 88, 该探针同样对 $\mathrm{H}_{2} \mathrm{~S}$ 表现出高灵 敏度高选择性响应, 而其它生物硫醇物质不会产生此响 应.

Nagano 等 ${ }^{[79]}$ 基于氮杂大环 $\mathrm{Cu}^{2+}$ 配合物合成得到一 种新型 $\mathrm{H}_{2} \mathrm{~S}$ 探针 89 (Eq. 34). 该探针可以在水溶液中高 选择性的识别 $\mathrm{H}_{2} \mathrm{~S}$, 具有极强的光学性能和生物应用前 景. 研究表明, 该探针不仅可用于容器中 $\mathrm{H}_{2} \mathrm{~S}$ 的酶促释 放程度, 而且也用于活细胞中 $\mathrm{H}_{2} \mathrm{~S}$ 的实时成像. 该探针 独特的性能优势有望进一步应用于 $\mathrm{CBS}$ (胱硫醚 $\beta$-合 酶), CSE(胱硫醚 $\gamma$-裂解酶)和 3MST (3-颈基丙酮酸硫基 转移酶)激动剂和拮抗剂的高效筛选及 $\mathrm{H}_{2} \mathrm{~S}$ 生物功能的 拓展研究.

2012 年, Zeng 等 ${ }^{[80]}$ 报道了一种 8-差基喹啉相连的 荧光素衍生物 90 (Eq. 35), 该探针与 $\mathrm{Cu}^{2+}$ 结合后, 可特 异性的识别 $\mathrm{S}^{2-}$. 在众多的阴离子中, 只有 $\mathrm{S}^{2-}$ 能使 $\mathrm{Cu}^{2+}$ 所淬灭的苂光重新恢复, 产生 “off-on” 型苂光响应, 实现对 $\mathrm{Cu}^{2+}$ 和 $\mathrm{S}^{2-}$ 的反复识别响应, 具有检测的可逆性.

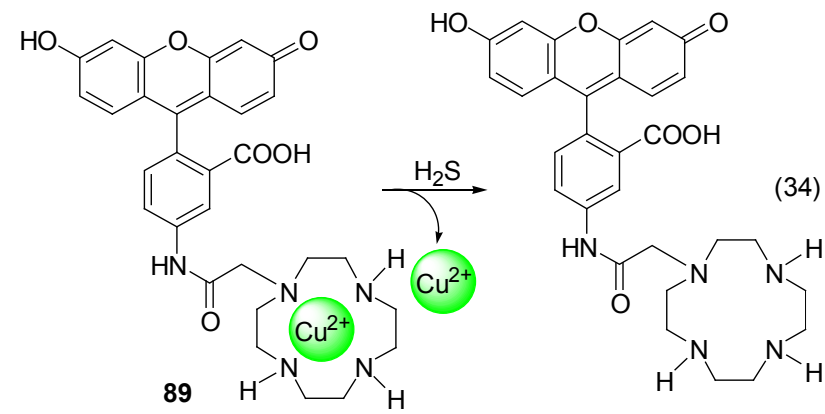

当向探针溶液中加入 $\mathrm{Cu}^{2+}$ 时, 将会使探针的紫色颜色 和绿色苂光消失而呈现黄色溶液, 向该黄色溶液中加入 $\mathrm{S}^{2-}$ 将又会使溶液变为紫色, 并产生绿色荧光. 此外, 生 物成像实验表明该探针可用于生物样品中低浓度 $\mathrm{S}^{2-}$ 的 检测.

\section{4 其它阴离子探针}

2010 年, Yoon 等 ${ }^{[81]}$ 报道了一种可以在水溶液中检 测 PPi 的识别体系, 该体系是基于罗丹明衍生物 91 和 $\mathrm{Al}^{3+}$ 所形成的配合物(Eq. 36). 91 一般处于无色无苂光状 态, $\mathrm{Al}^{3+}$ 的加入将使 91 发生金属诱导的内酰胺开环反应 而产生颜色和荧光变化. 而向该体系中加入焦磷酸盐 (PPi)将会使探针发生苂光淬灭并伴随着颜色的消失, 这 可能是由于 $\mathrm{Al}^{3+}$ 从体系中分离而使罗丹明回到闭环状 


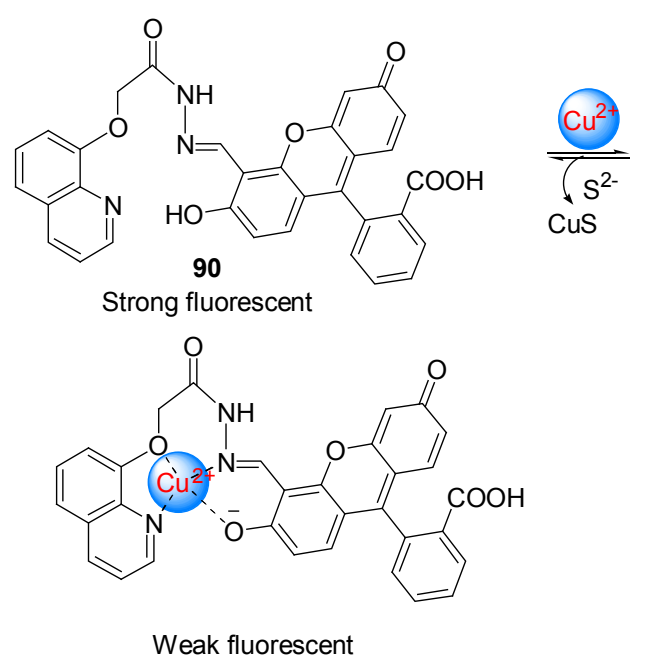

(35)

态，这已通过加入 PPi 前后 91- $\mathrm{Al}^{3+}$ 体系的 ESI-MS 谱图 证实. 该体系可用作中性水溶剂条件下 PPi 的检测, 且 不受其它生物竞争粒子如 AMP, ADP, ATP 和磷酸盐的 干扰.

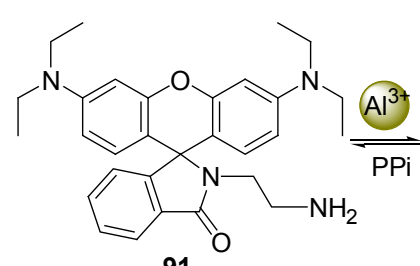

91

Colorless, non-fluorescent

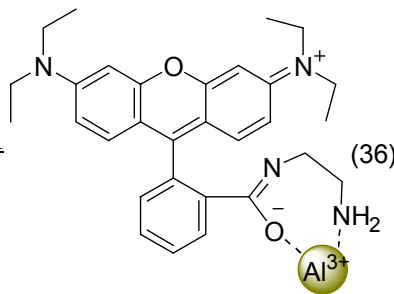

Pink, strong fluorescent
2011 年, Yang 等 ${ }^{[82]}$ 合成了一种含有醛基功能团的 罗丹明基苂光探针 92 (Eq. 37), 该探针可用于 EtOH$\mathrm{H}_{2} \mathrm{O}$ 体系中 $\mathrm{HSO}_{3}^{-}$的检测. 在诸多干扰离子环境下, 探 针 92 对 $\mathrm{HSO}_{3}^{-}$表现出较好的选择性, 可在 $1.0 \sim 50.0$ $\mu \mathrm{mol} / \mathrm{L}$ 浓度范围内实现对 $\mathrm{HSO}_{3}^{-}$的可逆性检测，是一 种性能优良的比色菼光探针, 具有进一步应用于实际样 品中 $\mathrm{HSO}_{3}^{-}$检测的潜在能力.

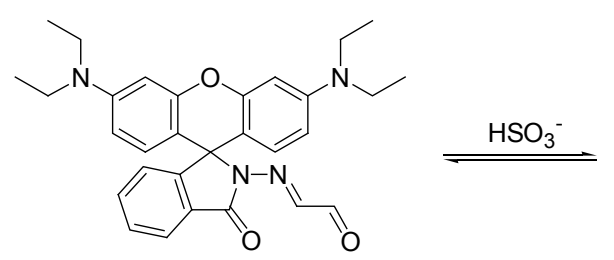

92<smiles>CCN(CC)c1ccc2c(-c3ccccc3C(=O)N/N=C/C(O)C(=O)O[Na])c3ccc(=[N+](CC)CC)cc-3oc2c1</smiles>

2012 年, Chatterjee 等 ${ }^{[83}$ 报道了一种可以检测水溶 液中 $\mathrm{NO}_{2}{ }^{-}$的探针 93 (Scheme 25), 该检测是通过酸性条
件下氨基的重氮化反应来实现的. 该探针对 $\mathrm{NO}_{2}{ }^{-}$表现 出独特的选择性，通过诱导的水解分离生成罗丹明 $\mathrm{B}$ 而 产生菼光. 探针 93 可在较快的时间内实现饱和，具有一 定的灵敏度，检测限可达 $0.13 \mu \mathrm{mol} / \mathrm{L}$ ，其应用前景广 阔。<smiles>CCN(CC)c1ccc2c(c1)Oc1cc(N(CC)CC)ccc1C21c2ccccc2C(=O)N1c1ccccc1</smiles>

93<smiles>CCN(CC)c1ccc2c(c1)Oc1cccc(N(CC)CC)c1C21c2ccccc2C(=O)N1c1ccccc1[NH3+]</smiles><smiles>CCN(CC)c1ccc2c(-c3ccccc3C(=O)n3nnc4ccccc43)c3ccc(=[N+](CC)CC)cc-3oc2c1</smiles><smiles>CCN(CC)c1ccc2c(-c3ccccc3C(=O)O)c3ccc(=[N+](CC)CC)cc-3oc2c1</smiles>

Scheme 25

\section{4 总结和展望}

近年来各种不同检测平台的荧光探针合成设计取 得了非凡的进展，而氧杂蒽及其衍生物内酰胺(或内酯) 开环反应为设计新型探针提供了重要的模式. 本文系统 综述了近年来基于氧杂葸及其衍生物探针对阳离子、 $\mathrm{pH}$ 、阴离子的检测, 并对探针的发光机理及其在生物成 像方面的应用进行了详细的讨论. 对于罗丹明 B 和罗丹 明 $6 \mathrm{G}$ 衍生物, 其结构改进主要集中在酰胺 $\mathrm{N}$ 原子部分, 以及其衍生结构的优化修饰. 相比之下，苂光素和罗丹 明 110 衍生物的改进只要集中在氧杂葱环上的差基和氨 基上，在目标分子作用下发生断键反应而释放出酚羟基 和氨基，进而使内酯隐色基团开环产生苂光.

归纳和分析近年来的文献报道，目前对于氧杂蒽苂 光探针研究呈现如下趋势: (1)响应方式由单一信号向多 信号响应发展; (2)研究内容由识别检测应用向识别机理 研究发展; (3)检测对象由非生命体向生命活体方向发 展. 在众多探针中, Schiff 碱识别团以其独特且优良的 识别性能受到了很大的关注，是设计新型探针不可忽略 的识别团. 而将其它苂光团或量子点与氧杂蒽相连所得 
到的 FRET 和 TBET 型探针也为高性能荧光探针的发展 提供了较大的推进. 此外, 将氧杂葱衍生物和纳米材料 或高分子聚合物串连也为环境中金属离子的快速有效 检测提供了良好的参考. 近年来新型六元螺环隐色体探 针的出现则将氧杂葱型荧光探针的发展提升到另一个 层面. 相比于传统的五元螺环隐色体探针, 六元螺环探 针具有很好的潜在结构优势, 可极大的改善探针的选择 性、稳定性和荧光性能, 将会成为今后氧杂葸型苂光探 针发展的一个小分支. 因此, 对于氧杂葱及其衍生物片 段的结构调控和性能优化仍是一个具有挑战性和发展 潜力的课题, 在生物识别及环境检测等领域有着广阔的 拓展空间和应用前景.

\section{References}

[1] Jung, H. S.; Chen, X. Q.; Kim, J. S.; Yoon, J. Chem. Soc. Rev. 2013, 42, 6019.

[2] Yang, Y. M.; Zhao, Q.; Feng, W.; Li, F. Y. Chem. Rev. 2013, 113, 192.

[3] Wang, Y. G.; Liu, Y. Chemical Labels and Probe Technology in the Application of Molecular Biology, Chemical Industry Press, Beijing, 2007, pp. $1 \sim 5$ (in Chinese).

(王彦广, 刘洋, 化学标记与探针技术在分子生物学中的应用, 化学工业出版社, 北京, 2007, pp. 1 5.)

[4] (a) Ramette, R. W.; Sandell, E. B. J. Am. Chem. Soc. 1956, 78, 4872 .

(b) Beija, M.; Afonso, C. A.; Martinho, J. M. Chem. Soc. Rev. 2009, 38, 2410.

[5] Dujols, V.; Ford, F.; Czarnik, A. W. J. Am. Chem. Soc. 1997, 119, 7386.

[6] (a) Kim, H. N.; Lee, M. H.; Kim, H. J.; Kim, J. S.; Yoon, J. Chem. Soc. Rev. 2008, 37, 1465.

(b) Chen, X. Q.; Tian, X. Z.; Shin, I.; Yoon, J. Chem. Soc. Rev. 2011, 40, 4783.

(c) Chen, X. Q.; Pradhan, T.; Wang, F.; Kim, J. S.; Yoon, J. Chem. Rev. 2012, 112, 1910.

[7] Li, N.; Liu, M. L.; Yin, W. T.; Yang, Z.; Shi, Z. Chin. J. Org. Chem. 2011, 31, 39 (in Chinese).

(李娜, 刘美玲, 尹文婷, 杨征, 李剑利, 史真, 有机化学, 2011, 31,39.)

[8] He, H. Z.; Lei, L.; Li, J. L.; Shi, Z. Chin. J. Chem. 2009, 27, 1169.

[9] Wu, L. X.; Dai, Y. R.; Marriott, G. Org. Lett. 2011, 13, 2018.

[10] Collot, M.; Loukou, C.; Yakovlev, A. V.; Wilms, C. D.; Li, D. D.; Evrard, A.; Zamaleeva, A.; Bourdieu, L.; Leger, J. F.; Ropert, N.; Eilers, J.; Oheim, M.; Feltz, A.; Mallet, J. M. J. Am. Chem. Soc. 2012, 134, 14923

[11] (a) Zhou, Y.; Wang, F.; Kim, Y.; Kim, S. J.; Yoon, J. Org. Lett. 2009, 11,4442 .

(b) Zhao, Y.; Zhang, X. B.; Han, Z. X.; Qiao, L.; Li, C. Y.; Jian, L. X.; Shen, G. L.; Yu, R. Q. Anal. Chem. 2009, 81, 7022.

(c) Swamy, K. M. K.; Ko, S. K.; Kwon, S. K.; Lee, H. N.; Mao, C.; Kim, J. M.; Lee, K. H.; Kim, J.; Shin, I.; Yoon, J. Chem. Commun. 2008, 5915.

(d) Huang, L.; Wang, X.; Xie, G. Q.; Xi, P. X.; Li, Z.; Xu, M. P.; Wu, Y. J.; Bai, D. C.; Zeng, Z. Z. Dalton Trans. 2010, 39, 7894.

(e) Yu, F. B.; Zhang, W. S.; Li, P.; Xing, Y. L.; Tong, L. L.; Ma, J. P.; Tang, B. Analyst 2009, 134, 1826.

(f) Chen, X. Q.; Jou, M. J.; Lee, H.; Kou, S.; Lim, J.; Nam, S. W.;
Park, S.; Kim, K. M.; Yoon, J. Sens. Actuators, B: Chem. 2009 137, 597.

(g) Xi, P. X.; Dou, J. Y.; Huang, L.; Xu, M.; Chen, F. J.; Wu, Y. J.; Bai, D. C.; Li, W. G.; Zeng, Z. Z. Sens. Actuators, B: Chem. 2010, $148,337$.

(h) Huang, L.; Chen, F. J.; Xi, P. X.; Xie, G. Q.; Li, Z. P.; Shi, Y. J.; Xu, M.; Liu, H. Y.; Ma, Z. R.; Bai, D. C.; Zeng, Z. Z. Dyes Pigm. 2011, 90, 265.

(i) Huo, F. J.; Yin, C. X.; Yang, Y. T.; Su, J.; Chao, J. B.; Liu, D. S. Anal. Chem. 2012, 84, 2219.

(j) Wang, J. L.; Lomh, L. P.; Xie, D.; Song, X. F. Sens. Actuators, B: Chem. 2013, 177, 27.

(k) Maity, D.; Karthugeyan, D.; Kumdu, T. K.; Govindaraju, T. Sens. Actuators, B: Chem. 2013, 176, 831.

[12] Zhang, J. F.; Zhou, Y.; Yoon, J.; Kim, Y.; Kim, S. J.; Kim, J. S. Org. Lett. 2010, 12, 3852.

[13] Liu, W. Y.; Li, H. Y.; Zhao, B. X.; Miao, J. Y. Org. Biomol. Chem. 2011, 9, 4802.

[14] Yin, J. X.; Ma, X.; Wei, G. H.; Wei, D. B.; Du, Y. G. Sens. Actuators, B: Chem. 2013, 177, 213.

[15] Yang, Z.; She, M. Y.; Zhang, J.; Chen, X. X.; Huang, Y. Y.; Zhu, H. Y.; Liu, P.; Li, J. L.; Shi, Z. Sens. Actuators, B: Chem. 2013, $176,482$.

[16] Yuan, L.; Lin, W. Y.; Chen, B.; Xie, Y. N. Org. Lett. 2012, 14, 432.

[17] Wu, C.; Bian, Q. N.; Zhang, B. G.; Cai, X.; Zhang, S. D.; Zheng, H.; Yang, S. Y.; Jiang, Y. B. Org. Lett. 2012, 14, 4198.

[18] Fan, J. L.; Zhan, P.; Hu, M. M.; Sun, W.; Tang, J. Z.; Wang, J. Y.; Sun, S. G.; Song, F. L.; Peng, X. J. Org. Lett. 2013, 15, 492.

[19] (a) Suresh, M.; Mishra, S.; Mishra, S. K.; Suresh, E.; Mandal, A. K.; Shrivastav, A.; Das, A. Org. Lett. 2009, 11, 2740.

(b) Du, J. J.; Fan, J. L.; Peng, X. J.; Sun, P. P.; Wang, J. Y.; Li, H. L.; Sun, S. G. Org. Lett. 2010, 12, 476.

(c) Huang, W.; Zhu, X.; Wua, D. Y.; He, C.; Hu, X. Y.; Duan, C. Y. Dalton Trans. 2009, 10457.

(d) Suresh, M.; Shrivastav, A.; Mishra, S.; Suresh, E.; Das, A. Org. Lett. 2008, 10, 3013.

(e) Kim, H. N.; Nam, S. W.; Swamy, K. M. K.; Jin, Y.; Chen, X. Q.; Kim, Y.; Kim, S. J.; Park, S.; Yoon, J. Analyst 2011, 136, 1339.

(f) Huang, W.; Zhou, P.; Yan, W. B.; He, C.; Xiong, L. Q.; Li, F. Y.; Duan, C. Y. J. Environ. Monitor. 2009, 11, 330.

(g) Tang, L. J.; Li, F. F.; Liu, M. H.; Nandhakumar, R. Spectrochim. Acta A 2011, 78, 1168.

[20] Wu, D. Y.; Huang, W.; Duan, C. Y.; Lin, Z. H.; Meng, Q. J. Inorg. Chem. 2007, 46, 1538.

[21] Yang, H.; Zhou, Z. G.; Huang, K. W.; Yu, M. X.; Li, F. Y.; Yi, T.; Huang, C. H. Org. Lett. 2007, 9, 4729.

[22] (a) Wu, D. Y.; Huang, W.; Lin, Z. H; Duan, C. Y.; He, C.; Wu, S.; Wang, D. H. Inorg. Chem. 2008, 47, 7190.

(b) Huang, W.; Wu, D. Y.; Duan, C. Y. Inorg. Chem. Commun. 2010, 13, 294.

[23] Xi, P. X.; Huang, L.; Xie, G. Q.; Chen, F. J.; Xu, Z. H.; Bai, D. C.; Zeng, Z. Z. Dalton Trans. 2011, 40, 6382.

[24] Lee, Y. H.; Lee, M. H.; Zhang, J. F.; Kim, J. S. P. J. Org. Chem. 2010, 75, 7159 .

[25] Fang, G.; Xu, M. Y.; Zeng, F.; Wu, S. Z. Langmuir 2010, 26, 17764.

[26] Ma, C.; Zeng, F.; Huang, L. f.; Wu, S. Z. J. Phys. Chem. B 2011, $115,874$.

[27] Kumar, M.; Kumar, N.; Bhalla, V.; Singh, H.; Sharma, P. R.; Kaur, T. Org. Lett. 2011, 13, 1422.

[28] Bhalla, V.; Roopa; Kumar, M.; Sharma, P. R.; Kaur, T. Inorg. Chem. 2012, 51, 2150. 
[29] Gong, Y. J.; Zhang, X. B.; Zhang, C. C.; Luo, A. L.; Fu, T.; Tan, W. H.; Shen, G. L.; Yu, R. Q. Anal. Chem. 2012, 84, 10777.

[30] Wang, W.; Li, Y. P.; Sun, M. D.; Zhou, C.; Zhang, Y.; Li, Y. X.; Yang, Q. B. Chem. Commun. 2012, 48, 6040.

[31] Zhang, D.; Li, M.; Wang, M.; Wang, J. H.; Yang, X.; Ye, Y.; Zhao, Y. F. Sens. Actuators, B: Chem. 2013, 177, 997.

[32] Yang, Z.; Hao, L. K.; Yin, B.; Obst, M.; Kappler, A.; Li, J. L. Org. Lett. 2013, 15, 4334.

[33] Han, Z. X.; Zhang, X. B.; Li, Z.; Gong, Y. J.; Wu, X. Y.; Jin, Z.; He, C. M.; Jian, L. X.; Zhang, J.; Shen, G. L.; Yu, R. Q. Anal. Chem. 2010, 82, 3108.

[34] Iyoshi, S.; Taki, M.; Yamamoto, Y. Org. Lett. 2011, 13, 4558.

[35] Xu, L.; Xu, Y. F.; Zhu, W. P.; Zeng, B. B; Yang, C. M.; Wu, B.; Qian, X. H. Org. Biomol. Chem. 2011, 9, 8284.

[36] Sreenath, K.; Clark, R. J.; Zhu, L. J. Org. Chem. 2012, 77, 8268.

[37] Ju, H.; Lee, M. H.; Kim, J.; Kim, J. S.; Kim, J. Talanta 2011, 83, 1359.

[38] Tyagi, A. K.; Ramkumar, J.; Jayakumar, O. D. Analyst 2012, 137, 760.

[39] Wang, S. X.; Meng, X. M.; Zhu, M. Z. Tetrahedron Lett. 2011, 52, 2840.

[40] Chereddy, N. R.; Thennarasu, S.; Mandal, A. B. Dalton Trans. 2012, 41, 11753.

[41] Huang, L.; Hou, F. P.; Cheng, J. Xi, P. X.; Chen, F. L.; Bai, D. C.; Zeng, Z. Z. Org. Biomol. Chem. 2012, 10, 9634.

[42] Weerasinghe, A. J.; Schmiesing, C.; Varaganti, S.; Ramakrishna, G.; Sinn, E. J. Phys. Chem. B 2010, 114, 9413.

[43] Bhalla, V.; Sharma, N.; Kumar, N.; Kumar, M. Sens. Actuators, B: Chem. 2013. 178, 228.

[44] Zheng, X. Y.; Zhang, W. J.; Mu, L.; Zeng, X.; Xue, S. F.; Tao, Z.; Yamatob, T. J. Inclusion Phenom. Macrocyclic Chem. 2010, 68, 139.

[45] Ma, B. L.; Wu, S. Z.; Zeng, F.; Luo, Y. L.; Zhao, J. Q.; Tong, Z. Nanotechnology 2010, 21, 195501/1.

[46] Ma, B. L.; Wu, S. Z.; Zeng, F. Sens. Actuators, B: Chem. 2010, $145,451$.

[47] Wang, B. D.; Hai, J.; Liu, Z. C.; Wang, Q.; Yang, Z. Y.; Sun, S. H. Angew. Chem., Int. Ed. 2010, 49, 4576.

[48] (a) Yang, Z.; She, M. Y.; Yin, B.; Cui, J. H.; Zhang, Y. Z.; Sun, W.; Li, J. L.; Shi, Z. J. Org. Chem. 2012, 77, 1143.

(b) She, M. Y.; Yang, Z.; Yin, B.; Zhang, J.; Gu, J.; Yin, W. T.; Li, J. L.; Zhao, G. F.; Shi, Z. Dyes Pigm. 2012, 92, 1337.

(c) Yin, W. T.; Cui, H.; Yang, Z.; Li, C.; She, M. Y.; Yin, B.; Li, J. L.; Zhao, G. F.; Shi, Z. Sens. Actuators, B: Chem. 2011, 157, 675.

[49] Chereddy, N. R.; Thennarasu, S.; Mandal, A. B. Analyst 2013, 138, 1334.

[50] Meng, Q. T.; Su, W. P.; Hang, X. M.; Li, X. Z.; He, C.; Duan, C. Y. D. Talanta 2011, 86, 408.

[51] Pu, S. Z.; Wang, T. F.; Liu, G.; Liu, W. J.; Cui, S. Q. Dyes Pigm. 2012, 94, 416.

[52] Mahato, P.; Saha, S.; Suresh, E.; Liddo, R. D.; Parnigotto, P. P.; Conconi, M. T.; Kesharwani, M. K.; Ganguly, B.; Das, A. Inorg. Chem. 2012, 51, 1769

[53] Chatterjee, A.; Santra, M.; Won, N.; Kim, S.; Kim, J. K.; Kim, S. B.; Ahn, K. H. J. Am. Chem. Soc. 2009, 131, 2040.

[54] Hu, M. M.; Fan, J. L.; Cao, J, F.; Song, K. D.; Zhang, H.; Sun, S. G.; Peng, X. J. Analyst 2012, 137, 2107.

[55] Yang, Y.; Lee, S.; Tae, J. Org. Lett. 2009, 11, 5610.

[56] (a) Jou, M. J.; Chen, X. Q.; Swamy, K. M. K.; Kim, H. N.; Kim, H.;
Lee, S.; Yoon, J. Chem. Commun. 2009, 7218.

(b) Egorova, O. A.; Seo, H.; Chatterjee, A.; Ahn, K.-H. Org. Lett. 2010, 12, 401.

[57] Seo, H.; Jun, M. E.; Egorova, O. A.; Lee, K. H.; Kim, K. T.; Ahn, K. H. Org. Lett. 2012, 14, 5062.

[58] Kim, H.; Lee, S.; Lee, J.; Tae, J. Org. Lett. 2010, 12, 5342.

[59] Jun, M. E.; Ahn, K. H. Org. Lett. 2010, 12, 279.

[60] Li, H. L.; Fan, J. L.; Du, J. J.; Guo, Ke. X.; Sun, S. G.; Liu, X. J.; Peng, X. J. Chem. Commun. 2010, 46, 1079.

[61] Li, H. L.; Fan, J. L.; Hu, M. M.; Cheng, G. H.; Zhou, D. H.; Wu, T.; Song, F. L.; Sun, S. G.; Duan, C. Y.; Peng, X. J. Chem.-Eur. J. 2012, 18, 12242 .

[62] Balamurugan, R.; Chien, C. C.; Wu, K. M.; Chiu, Y. H.; Liu, H. H. Analyst 2013, 138, 1564.

[63] Huang, W.; Wu, D. Y.; Guo, D.; Zhu, X.; He, C.; Meng, Q. J.; Duan, C. Y. Dalton Trans. 2009, 2081.

[64] Wang, Q.; Li, C. Y.; Zou, Y.; Wang, H. X.; Yi, T.; Huang, C. H. Org. Biomol. Chem. 2012, 10, 6740.

[65] Wu, S. Q.; Li, Z.; Han, J. H.; Han, S. F. Chem. Commun. 2011, 47, 11276.

[66] Shi, W.; Li, X. H.; Ma, H. M. Angew. Chem., Int. Ed. 2012, 51, 6432.

[67] Zhu, H.; Fan, J. L.; Xu, Q. L.; Li, H. L.; Wang, J. Y.; Gao, P.; Peng, X. J. Chem. Commun. 2012, 48, 11766.

[68] Liu, Y. L.; Sun, Y.; Du, J.; Lv, X.; Zhao, Y.; Chen, M. L.; Wang, P.; Guo, W. Org. Biomol. Chem. 2011, 9, 432.

[69] Yuan, L.; Lin, W. Y.; Xie, Y. N.; Chen, B.; Song, J. Z. Chem.-Eur. J. 2012, 18, 2700.

[70] Chen, G. W.; Song, F. L.; Wang, J. Y.; Yang, Z. G.; Sun, S. G.; Fan, J. L.; Qiang, X. X.; Wang, X.; Dou, B. R.; Peng, X. J. Chem. Commun. 2012, 48, 2949.

[71] Jin, X. L.; Hao, L. K.; Hu, Y. L.; She, M. Y.; Shi, Y. N.; Obst, M. Li, J. L.; Shi, Z. Sens. Actuators, B: Chem. 2013, 186, 56.

[72] Lv, X.; Liu, J.; Liu, Y. L.; Zhao, Y.; Chen, M. L.; Wang, P.; Guo, W. Org. Biomol. Chem. 2011, 9, 4954.

[73] Sumiya, S.; Doi, T.; Shiraishi, Y.; Hirai, T. Tetrahedron 2012, 68, 690.

[74] Liu, Y. L.; Lv, X.; Zhao, Y.; Liu, J.; Sun, Y. Q.; Wang, P.; Guo, W. J. Mater. Chem. 2012, 22, 1747.

[75] Jo, J.; Olasz, A.; Chen, C.; Lee, D. J. Am. Chem. Soc. 2013, 135, 3620.

[76] Yang, X. F.; Wang, L. P.; Xu, H. M.; Zhao, M. L. Anal. Chim. Acta 2009, 631, 91.

[77] Liu, C. R.; Pan, J.; Li, S.; Zhao, Y.; Wu, L. Y.; Berkman, C. E.; Whorton, A. R.; Xian, M. Angew. Chem., Int. Ed. 2011, 50, 10327.

[78] Liu, C. R.; Peng, B.; Li, S.; Park, C. M.; Whorton, A. R.; Xian, M. Org. Lett. 2012, 14, 2184.

[79] Sasakura, K.; Hanaoka, K.; Shibuya, N.; Mikami, Y.; Kimura, Y.; Komatsu, T.; Ueno, T.; Terai, T.; Kimura, H.; Nagano, T. J. Am. Chem. Soc. 2011, 133, 18003.

[80] Hou, F. P.; Huang, L.; Xi, P. X.; Cheng, J.; Zhao, X. F.; Xie, G. Q.; Shi, Y. J.; Cheng, F. J.; Yao, X. J; Bai, D. C.; Zeng, Z. Z. Inorg. Chem. 2012, 51, 2454.

[81] Lohani, C. R.; Kim, J. M.; Chung, S. Y.; Yoon, J.; Lee, K. H. Analyst 2010, 135, 2079.

[82] Yang, X. F.; Zhao, M. L.; Wang, G. Sens. Actuators, B: Chem. 2011, 152, 8 .

[83] Kumar, V.; Banerjee, M.; Chatterjee, A. Talanta 2012, 99, 610. 